\title{
Enhanced Multi-Objective Energy Optimization by a Signaling Method
}

\author{
João Soares ${ }^{1, *}$, Nuno Borges ${ }^{1}$, Zita Vale ${ }^{1}$ and P.B. de Moura Oliveira ${ }^{2}$ \\ 1 GECAD, Research Group on Intelligent Engineering and Computing for Advanced Innovation and \\ Development, Polytechnic of Porto, R. Dr. António Bernardino de Almeida, 431, Porto 4200-072, Portugal; \\ ndsbs@isep.ipp.pt (N.B.); zav@isep.ipp.pt (Z.V.) \\ 2 INESC Technology and Science, UTAD University, Quinta de Prados, Vila Real 5000-801, Portugal; \\ oliveira@utad.pt \\ * Correspondence: joaps@isep.ipp.pt; Tel.: +351-228-340-500; Fax: +351-228-321-159
}

Academic Editor: Chunhua Liu

Received: 2 August 2016; Accepted: 22 September 2016; Published: 10 October 2016

\begin{abstract}
In this paper three metaheuristics are used to solve a smart grid multi-objective energy management problem with conflictive design: how to maximize profits and minimize carbon dioxide $\left(\mathrm{CO}_{2}\right)$ emissions, and the results compared. The metaheuristics implemented are: weighted particle swarm optimization (W-PSO), multi-objective particle swarm optimization (MOPSO) and non-dominated sorting genetic algorithm II (NSGA-II). The performance of these methods with the use of multi-dimensional signaling is also compared with this technique, which has previously been shown to boost metaheuristics performance for single-objective problems. Hence, multi-dimensional signaling is adapted and implemented here for the proposed multi-objective problem. In addition, parallel computing is used to mitigate the methods' computational execution time. To validate the proposed techniques, a realistic case study for a chosen area of the northern region of Portugal is considered, namely part of Vila Real distribution grid (233-bus). It is assumed that this grid is managed by an energy aggregator entity, with reasonable amount of electric vehicles (EVs), several distributed generation (DG), customers with demand response (DR) contracts and energy storage systems (ESS). The considered case study characteristics took into account several reported research works with projections for 2020 and 2050. The findings strongly suggest that the signaling method clearly improves the results and the Pareto front region quality.
\end{abstract}

Keywords: electric vehicle (EV); emissions; energy resources management (ERM); multi-objective optimization; virtual power player (VPP); smart grid

\section{Introduction}

The increasing concern over global climate changes and air pollution has motivated policy makers to promote renewable energy sources [1]. Distributed generation (DG) based on renewable sources presents an opportunity to decrease this pollution impact considerably. The power industry represents a significant portion of the global carbon dioxide $\left(\mathrm{CO}_{2}\right)$ emissions corresponding to about $40 \%$ [2] and regulations are currently in place for controlling the level of emissions in this sector [1]. In Portugal the 2000s level of $\mathrm{CO}_{2}$ emissions from electricity generation was $480 \mathrm{kgCO}_{2} / \mathrm{MWh}$ [3]. In 2020 and 2050, it is expected that this level will drop to $190 \mathrm{~kg} \mathrm{CO} / \mathrm{MWh}$ and $20 \mathrm{kgCO}_{2} / \mathrm{MWh}$, respectively [3]. The emissions from power sector decline as more renewable based generation is integrated. This raises an interesting research issue which is discussed in this work: understanding the impact of considering $\mathrm{CO}_{2}$ emissions in the energy management problem up to 2050 within smart grids operation context.

With the emergence of intelligent infrastructures it will be possible to deploy smart grid capabilities, such as optimizing energy resources scheduling and reducing the load demand, 
avoiding high peaks at high electricity prices, while enabling a smoothed demand profile and profit maximization [4]. However, there may exist other challenges beyond maximizing the profit, such as minimizing $\mathrm{CO}_{2}$ emissions due to environmental concerns $[5,6]$. For that reason it is necessary to find an optimal solution that considers two or more objectives. Hence, in most real-world circumstances system operators are faced with a multi-objective problem [7-10].

In the literature, several approaches have been reported concerning intelligent energy resources management (ERM) [8,9,11-17]. Regarding ERM under different design goals, several multi-objective models have been investigated so far, to determine the optimal resource scheduling of a microgrid over a day-ahead period time. In [16], the proposed ERM optimizes an aggregated multi-objective criterion taking into account both the energy cost and thermal comfort of the occupants. In most multi-objective ERM models developed in the literature, minimizing the emissions is considered as an additional objective [8,9,11-15]. The multi-objective model published in [15] does not include the power flow equations, electric vehicles (EVs), demand response (DR) and energy storage systems (ESS). The model proposed in [11] considers a simple load balance (active power) and does not consider the presence of EVs neither any type of DR. EVs and DR resources can bring important benefits to the future smart grid $[14,18]$. A Benders' decomposition approach to solve ERM is presented in [8]. This work proposes a multi-objective operational scheduling for charging and discharging of EVs in a smart distribution network in the day-ahead context. The multi-objective optimization considers both costs and emissions but the work seems limited since it does not formulate or consider demand response, wind or photovoltaic (PV) generation and ESS, which are vital smart grids features. Moreover, uncertainties of the day-ahead decisions are not addressed. In [17], a differential search algorithm (DSA) and quantum particle swarm optimization (QPSO) are used to solve a hard combinatorial scheduling problem. A comparison between the DSA and QPSO, with their hybrid versions is presented. However, that work considers only a single objective function to maximize the profits and not addressing DR, ESS, market bids (sell or buy), and $\mathrm{CO}_{2}$ emissions. In [19], the tradeoff between cost and environment emissions is presented using a regular and a binary particle swarm optimization (PSO). The computational intelligence-based scheduling seems promising to reduce cost and emissions, while maximizing the renewable energy sources use. The multi-objective problem is solved using a weighted sum approach with PSO, instead of a multi-objective evolutionary algorithm or multi-objective PSO (MOPSO) [20]. Moreover, the network constraints are not considered in the mentioned approach. In [15], a multi-objective energy management for a micro-grid using both intelligent techniques and linear programming is presented to minimize the operation costs and the environment impacts. However, the work proposes a linear formulation without power flow equations and not considering the possibility of vehicle-to-grid (V2G). A modified PSO methodology is developed in $[21,22]$ to solve the problem of ERM with high penetration of DG and EVs with V2G, with the aim to improve the performance of PSO. However, the reported work considers a single objective function, i.e., the operation cost minimization. In [18], a multi-objective model is presented, introducing the ERM model reliability concern through a multi-objective formulation. Pareto solutions provide multiple alternatives for the energy aggregator, namely by picking the most appropriate solution, taking into account the preference between minimum operation cost and the maximum available reserve. Weighted PSO (W-PSO) is proposed to solve the multi-objective problem, which uses a Pareto set scheme.

The problem handled in this paper concerns a profit seeking aggregator entity managing several distributed energy resources (DER). The aggregator needs to solve the ERM problem, which is a large-scale non-linear combinatorial DER scheduling problem including V2G resources, DR, sells/buys market and external suppliers. A multi-objective function is used to maximize the profit corresponding to the difference between the income and operating costs and a function to minimize the $\mathrm{CO}_{2}$ emissions. Three metaheuristics are compared, namely, the W-PSO, MOPSO and non-dominated sorting genetic algorithm II (NSGA-II). Hence, the previous signaling method for PSO used in [23] is adapted and used here to help W-PSO, MOPSO and NSGA-II to escape violations and improve fitness function. That 
way, this paper reviews the signaling method influence in the multi-objective problem. This validation has not been addressed before in the literature, so it constitutes its major contribution.

The work is validated with a case study concerning a real 233-bus distribution network from a region of northern Portugal, namely a part of the distribution grid from Vila Real city. Two scenarios are assessed and evaluated using the multi-objective approaches for years 2020 and 2050, respectively. In addition to the renewable generation increase from 2020 to 2050, EVs are expected to be increasingly adopted. The study presented here takes into account these changes, including different DG technologies EVs characteristics. The electricity grid is slightly updated, namely expanding the DG units' capacity to supply approximately 14,000 consumers. The EVs scenarios reach 5080 EVs in 2050, enabled with V2G capability. The results obtained suggest that signaling method effectively improves Pareto front in the tested metaheuristics and finds profitable solutions with less $\mathrm{CO}_{2}$ impact and without constraint violations. Furthermore, W-PSO seems to present better results, but requiring more computational times. The robustness test and sensitivity analysis suggest that signaling method is robust and works well under variations of important parameters.

This paper is organized as follows: after this introductory part, Section 2 presents the multi-objective ERM mathematical model, Section 3 presents the technical solutions employed in this paper, Section 4 discusses the case study, and finally Section 5 presents our conclusions.

\section{Multi-Objective Energy Resource Management}

In this section the mathematical model of the day-ahead energy resource management problem for virtual power players (VPPs) is presented. VPP is an energy aggregator entity that aggregates several types of energy sources, namely DG units, and it is responsible for managing them using a set of sophisticated software tools in order to raise their value and competitiveness. A VPP is defined in [24] as "a system that relies upon software systems to remotely and automatically dispatch and optimize generation, demand-side, or storage resources (including EVs and bidirectional inverters) in a single, secure web-connected system". VPPs are well-studied and defined in the literature, so the reader can refer to [25-27] to find more information about these entities.

\subsection{Mathematical Model}

The envisaged problem is a hard combinatorial mixed-integer non-linear programming (MINLP) problem due to multi-periodicity, high number of continuous, discrete, and binary variables and network non-linear equations. The VPP two conflicting objectives are: to maximize profits, as shown in Equation (1), and to minimize the $\mathrm{CO}_{2}$ emissions, as shown in Equation (2):

$$
\begin{aligned}
& \text { Minimize Profit }=O C-\text { In } \\
& \text { Minimize Emissions = Em }
\end{aligned}
$$

The VPP can receive its income (In) from four sources, as seen in Equation (3): the consumers demand revenue, the energy sold to the electricity market or to other entities, the revenue from the charging process of storage units and from the EVs charging:

$$
\begin{aligned}
& \text { Maximize In }= \\
& \sum_{t=1}^{T}\left[\left(\begin{array}{l}
\sum_{L=1}^{N_{L}} P_{\text {Load }(L, t)} \cdot M P_{\text {Load }(L, t)}+\sum_{M=1}^{N_{M}} P_{\text {Sell }(M, t)} \cdot M P_{\text {Sell }(M, t)}+ \\
\sum_{E=1}^{N_{E}} P_{\text {Charge }(E, t)} \cdot M P_{\text {Charge }(E, t)}+\sum_{V=1}^{N_{V}} P_{\text {Charge }(V, t)} \cdot M P_{\text {Charge }(V, t)}
\end{array}\right)\right]
\end{aligned}
$$

Function operation costs $(O C)$ in Equation (4) represents the operation cost of the resources managed/contracted by the VPP. It considers the cost with DG, external suppliers, discharge 
of ESS and EVs, DR, penalization with non-supplied demand, and penalization with DG units' generation curtailment:

$$
\begin{aligned}
& \text { Minimize } O C= \\
& \sum_{t=1}^{T}\left[\left(\begin{array}{l}
\sum_{I=1}^{N_{D G}} P_{D G(I, t)} \cdot c_{D G(I, t)}+\sum_{S=1}^{N_{S}} P_{\text {Supplier }(S, t)} \cdot c_{\text {Supplier }(S, t)}+ \\
\sum_{L=1}^{N_{L}} P_{\operatorname{Load} R R(L, t)} \cdot c_{\text {LoadDR }(L, t)}+\sum_{E=1}^{N_{E}} P_{\text {Discharge }(E, t)} \cdot c_{\text {Discharge }(E, t)}+ \\
\sum_{V=1}^{N_{V}} P_{\text {Discharge }(V, t)} \cdot c_{\text {Discharge }(V, t)}+\sum_{L=1}^{N_{L}} P_{N S D(L, t)} \cdot c_{N S D(L, t)}+ \\
\sum_{I=1}^{N_{D G}} P_{G C P(I, t)} \cdot c_{G C P(I, t)}
\end{array}\right)\right]
\end{aligned}
$$

Equation (5) shows the objective function to minimize the $\mathrm{CO}_{2}$ emissions:

$$
\begin{aligned}
& \text { Minimize } E m= \\
& \sum_{t=1}^{T}\left[\left(\begin{array}{l}
\sum_{D G}^{\Omega_{I=1}^{d}} P_{D G(I, t)} \times E_{D G(I, t)}+ \\
\Omega_{S P}^{e} \\
\sum_{S=1}^{e} P_{\text {Supplier }(S, t)} \times E_{\text {Supplier }(S, t)}
\end{array}\right)\right]
\end{aligned}
$$

\subsection{Model Constraints}

The problem constraints are similar to those given in [17]. The problem is mainly constrained by the network equations, namely active and reactive powers, voltage and angle limits, DG generation and supplier limits in each period, ESS capacity, charge and discharge rate limits, EVs capacity, EVs' trips requirements, charge and discharge rate limits (see Appendix A).

In the AC power flow model, both voltage magnitude and angle limits are considered. The maximum and minimum limits for the voltage angle are constrained between $[-\pi, \pi]$, while the magnitude can vary $\pm 5 \%$. The constraints for high-voltage (HV)/medium-voltage (MV) power transformers limits considering the power flow direction from HV to MV are taken into account, where the maximum amount of apparent power supplied from the upstream network cannot be higher than a predefined limit. This limit can correspond in reality to the capacity of the substation [23]. In the present ERM model the upstream network supply corresponds to the power bought from the external suppliers. For the three metaheuristics implemented in this paper, a full AC power flow is used [28] to check the network conditions. The efficient load flow solution uses the forward and backward propagation to calculate branch currents and node voltages [28]. With this information, it is possible to evaluate if the metaheuristics solutions violate the network constraints. The problem's mathematical constraints are detailed further in the Appendix A of this paper.

\subsection{Uncertainties}

Forecasts are very important to the vast majority of electricity players. However, they constitute imperfect information, due to forecasting errors. Certain input parameters in the proposed optimization model are not deterministic, e.g., the load forecast, and can change significantly after the optimization has been made. This is particularly true in day-ahead forecasts, with a larger time horizon. It is easier to understand that near real-time optimization, decisions are less prone to suffer from uncertainty issues in the ERM problem, mainly because forecasts' accuracy is much higher and faster decisions have to be made [29]. There are a few methods that can be deployed to handle uncertainties in these inputs. Two-stage stochastic programming is a suitable tool to handle scenario-based approaches, where a set of representative scenarios, which account for the expected variability of uncertain variables, are supplied to the two-stage optimization model. These models can provide a solution that takes into account the possible scenarios and their probability of occurrence, while minimizing a given expected 
cost. The drawback of such approach is that it requires one to know the error distribution model concerning the uncertain parameters, in order to build accurate scenarios. These scenarios must be well picked up in order to provide an accurate representation. Sometimes these distributions are not available, because there is a lack of historical data or missing information. Therefore, robust or fuzzy models can be used as an alternative to stochastic programming [30]. Robust optimization models are good for the analysis of the worst-case scenario. A solution may be called robust if it remains feasible and the objective function slightly changes as the inputs vary. These uncertainty parameters fall between a predefined range and the optimization seeks to find a solution that choses the best worst case situation, i.e., better payoff.

Uncertainty has received research efforts in single-objective context but few reported studies address it with a multiple criteria setting [31]. To handle input uncertainties, this paper uses the same approach as presented in [18], namely fuzzy theory applied to load and EVs demand forecasts. Furthermore, in the present multi-objective model, there are some types of DERs that can provide some flexibility and help to mitigate uncertainty coming from renewables when approaching real-time, namely ESS and DR resources. These DERs can be rescheduled in order to overcome the imbalances in the actual generation and consumption when approaching real-time. Nevertheless, this paper focus solely in the day-ahead model. For further details, real-time models have been addressed in [29,32,33].

\section{Technical Solutions}

Three technical solutions are presented to solve the multi-objective problem previously presented in Section 2. The methods implemented are: W-PSO, MOPSO and NSGA-II.

\subsection{Weighted Particle Swarm Optimization}

The particle swarm paradigm has been attracting the interest of researchers around the globe since its introduction in 1995 [9]. The initial ideas on particle swarms of Kennedy and Eberhart were essentially targeting analogues of social interaction, rather than purely individual cognitive abilities.

The particle swarm is a population-based stochastic algorithm for optimization which is based on social-psychological principles like flocks of birds or schools of fish. In PSO a number of particles are placed in the search space and each particle evaluates the objective function at its current location. Each particle keeps track of the coordinates associated with best solution found so far, this value is named of "personal best" (pbest). The particle also has access to information on the best solution found so far by others, this value is called "global best" (gbest). The basic idea of PSO is to accelerate every particle in the direction for the local of pbest and gbest. The value of acceleration varies randomly during the search procedure. While searching each particle uses the information from its best position in the past and the current best position among its neighbors. The movement is determined from the combination linear vectors with different weights. Each particle tries to change its position in accordance with the following information: current position, current speed, distance between the current position and personal best, distance between the current position and global best.

The present W-PSO implementation uses mutation of the strategic weights parameters [8]. At the beginning of the process, the values of the strategic parameters are randomly generated between 0 and 1. After that, the particle's weights are changed in each iteration using a Gaussian mutation distribution according to [8]. The velocity limits, the new particle's velocity and the new positions for each particles they are also calculated according to the well-recognized movement equation [8].

The weighted sum method $[18,34]$ is used in this context, to tackle the envisaged optimization problem. In this case, several runs with different weights for each objective function are evaluated before determining the Pareto front. The number of runs should take into account the tradeoff between the quality of the Pareto front and the necessary computational time. A parallel computing platform can mitigate this issue [18], used in this study to reduce the W-PSO computational burden. 
The fitness function in Equation (6) in W-PSO considers the total profit/loss, which is achieved by subtracting the $O C$ in Equation (4) to the income (In) in Equation (3), and the total emissions of $\mathrm{CO}_{2}$ $(E m)$, as follows:

$$
\text { fitness }=\left[\frac{(O C-I n)}{s_{1}} \times p w_{1}-\frac{s_{2}}{E m} \times p w_{2}\right]+\text { penalties }
$$

where:

$\begin{array}{ll}p w_{1} & \text { is the weight concerning the maximization of total profit } \\ p w_{2} & \text { is the weight concerning the minimization of total emissions } \\ \text { penalties } & \text { is the sum of penalties associated with solution's violations } \\ s_{1} & \text { is the normalization factor of profit } \\ s_{2} & \text { is the normalization factor of emissions }\end{array}$

The Pareto weights $p w_{1}$ and $p w_{2}$ are applied to the profit and the $\mathrm{CO}_{2}$ emissions, respectively. When $p w_{1}$ tends to $1\left(p w_{2} \rightarrow 0\right)$, the optimization will give more importance to maximize the profit. In the opposite case, when $p w_{1}$ tends to $0\left(p w_{2} \rightarrow 1\right)$, the optimization will give more importance to minimize the total emissions of $\mathrm{CO}_{2}$. Parameters $s_{1}$ and $s_{2}$ are normalization factors that must be adjusted for the optimization problem. The term penalties correspond to the violations detected in the evaluation phase. The penalties configured in W-PSO are the following: 100 for voltage limits violations, 1000 for line limits violations and 1000 for the solutions with insufficient generation. A full AC power flow is used [28] to check the network conditions.

\subsection{Multi-Objective Particle Swarm Optimization}

MOPSO is an advanced optimization algorithm to solve multi-objective problems [20] used in this work to handle the proposed energy management problem. MOPSO adopts an external repository and uses a mutation operator aiming to explore the remote region of the search space and the full range of each decision variable. A mutation of the strategic parameters as seen in the evolutionary PSO [35] has been adapted to MOPSO in this work, instead of the usual fixed parameters as in the original MOPSO [20]. This modification improved the cover rate and the overall front of the non-dominated solutions (NDS) as higher exploratory properties were introduced in the search procedure. Two types of mutation have been implemented in this work, which happen during the search loop, namely mutation of the parameters of the velocity equation and mutation in the position of some particles (randomly selected). The algorithm stops after the defined number of iterations is reached. This setup is widely used in other multi-objective metaheuristic-based algorithms [20,23,36,37].

The fitness function in Equation (7) in MOPSO considers the total profit and the emissions of $\mathrm{CO}_{2}$ :

$$
\text { fitness }=[(O C-I n)+\text { penalties } ; E m+\text { penalties }]
$$

In fact, the fitness in this case is a vector of values resulting from the several objective functions of the multi-objective problem (in this case two objectives), plus the penalties term, which corresponds to the sum of the penalty factors of the violations found a given individual during the evaluation stage. The penalties configured in MOPSO are the same as in W-PSO.

\subsection{Non-Dominated Sorting Genetic Algorithm II}

The NSGA-II proposed by Deb et al. in 2002 [38] has been applied to various problems and is considered one of the champions in multi-objective optimization [39]. NSGA-II has addressed some problems of the earlier proposal, NSGA, namely by reducing the high computational complexity of non-dominated sorting, from $O\left(m N^{3}\right)$ to $O\left(m N^{2}\right)$, introducing elitism and less parameters. The crossover and mutation operators remain as usual implemented in genetic algorithms, but selection operator works differently [40]. Selection is done with help of crowed-comparison operator, based on ranking, and crowding distance. Initially a random parent population is created. The population space 
is sorted based on the non-domination. Then each solution is assigned a fitness rank based on the non-domination level. The new generation is created using the tournament selection, crossover, and mutation. Elitism is introduced by comparing current population with the previously found best NDS. In the next step, parent and children are merged to form a new set of individuals and next generation is selected among this collection [34]. Like MOPSO, NSGA-II has the ability to find Pareto-optimal solutions in one single run. MOPSO and NSGA-II are selected as references techniques in this paper for multi-objective approaches due to their high relevance in the literature, namely in power systems problems. The fitness function in Equation (7), used in MOPSO is also implemented in NSGA-II.

\subsection{Multi-Dimensional Signaling Method}

In [23], a multi-dimensional signaling method is presented to improve metaheuristics performance to solve the energy scheduling problem. The method relies on a scheme to exchange signals between the metaheuristic core process, i.e., movement and evaluation stage. The signaling is important in both moments, namely identifying critical variables in the evaluation stage that should be treated differently in the movement stage. In the evaluation stage, the identification of violations also occurs. The following principles should be taken into account when deciding which variables should be signaled: if changed in a certain direction, variables that cannot be changed by direct repair method, variables that can improve fitness function.

In this case, the method is adapted to the multi-objective problem presented in this paper to help W-PSO, MOPSO, and NSGA-II escape violations and improve fitness function.

\subsubsection{Signaling Matrix Definition}

The multi-dimensional method uses a signaling matrix as a way to communicate between the evaluation and movement stage. The method is important in both moments, namely identifying critical variables in the evaluation stage that should be treated differently in the movement stage. In the evaluation stage, the signaling matrix changes if some conditions match the rules in the knowledge base. Later, in the movement stage, the signaling matrix is read and the signaled decision variables are mutated or changed. After the movement stage in each iteration the signaling matrix is reinitialized with zeros.

The multi-dimensional signaling matrix can be defined as $S^{p \times n} \in \mathbb{Z}^{p \times n}$ with $p$ elements (e.g., number of particles in PSO) and $n$ is the number of decision variables. The signal function presented in Equation (8), namely $\operatorname{sigFun}_{z}(S)$ and $\operatorname{sigFun}_{i}(S)$ changes the matrix $S$ values in each iteration at the evaluation stage if a violation of a given constraint $z$ is found, i.e., $g\left(c_{\mathrm{Z}}\left(x^{\mathrm{e}}\right)\right) \geq 0$, or if a custom condition $i\left(\vartheta_{i}\right)$ is true:

$$
S=\left\{\begin{array}{cc}
\operatorname{sigFun}_{z}(S), & \text { if } g\left(c_{z}\left(x^{e}\right)\right) \geq 0, z \in I, e \in p \\
\operatorname{sigFun}_{i}(S), & \text { if } \vartheta_{i}=\text { true }, i \in \mathbb{N}, i \neq z \\
0, & \text { otherwise }
\end{array}\right.
$$

where $z$ corresponds to the constraints that are suitable for signaling, $x$ to the $n$ decision variables and $\vartheta_{i}$ to the $i$ condition.

Variables which should be signaled during evaluation stage and their respective conditions, which identify the signaling, should be defined by the system implementer. The algorithm should be designed to evaluate the conditions and identify which variables should be signaled across iterations to improve solution fitness and/or constraint handling in the best way. The next section shows how signaling conditions have been implemented for the problem investigated in this paper.

\subsubsection{Implementation and Knowledge Base}

Signaling can be implemented for reducing constraints violations or improve fitness function. The most problematic constraints are related with network voltage, angles, and line thermal capacity. 
The appropriate variables are signaled to reduce or increase reactive/active power according to the type of violation in the respective zones, where a problem is found. Table 1 presents the signaling codes and rules to improve fitness function, i.e., improve profit, or reduce $\mathrm{CO}_{2}$ emissions. This signaling codes are applied by $\operatorname{sigFun}_{i}(S)$. In W-PSO the signaling may switch, in evaluation stage, between rules to improve profit (light grey) or reduce emissions (dark grey) according to the weights of the objective function (see Section 3.1, which refers to W-PSO weights scheme). In MOPSO and NSGA-II, the switching occurs according to a uniform random probability of $50 \%$. Yet, $P r_{s i g}$ is the global probability of signalling to be later applied in the movement phase, which is $80 \%$ in this case. Then an individual probability for each rule can be configured if desired, which influences the final probability of the signalling to really occur. It is important to remark that only $50 \%$ of the population individuals are selected to be signalled. The $M C_{(t)}$ represents the marginal cost of the system in period $t$.

Table 1. Signaling codes to improve fitness. EVs: electric vehicles; ESS: energy storage system; DR: demand response; and DG: distributed generation.

\begin{tabular}{|c|c|c|c|c|}
\hline Variables & Rule/Condition & Signal Code & Probability of Signal & Benefit \\
\hline \multirow{3}{*}{ EVs } & $M C_{(t)} \geq C_{\text {Discharge }(V, t)}$ & -1 & \multirow{2}{*}{$P r_{s i g}$} & \multirow{10}{*}{ Profit improve } \\
\hline & $M C_{(t)} \leq M P_{\text {Charge }(V, t)}$ & 1 & & \\
\hline & (Random) & 2 & $P r_{s i g} / 2$ & \\
\hline \multirow{3}{*}{ ESS } & $M C_{(t)} \geq C_{\text {Discharge }(E, t)}$ & -1 & \multirow{2}{*}{$P r_{s i g} / 2$} & \\
\hline & $M C_{(t)} \leq M P_{\text {Charge }(E, t)}$ & 1 & & \\
\hline & (Random) & 2 & $P r_{s i g} / 2$ & \\
\hline \multirow[b]{2}{*}{ DR } & $M C_{(t)} \geq\left(C_{\text {LoadDR }(L, t)}+M P_{\text {LoadDR }(L, t)}\right)$ & 1 & $P r_{\text {sig }}$ & \\
\hline & $M C_{(t)} \leq\left(C_{\text {Load } D R(L, t)}+M P_{\text {LoadDR }(L, t)}\right)$ & 2 & $P r_{\text {sig }}$ & \\
\hline \multirow{2}{*}{ Market sell } & 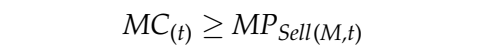 & 2 & $P r_{\text {sig }}$ & \\
\hline & $M C_{(t)} \leq M P_{S e l l(M, t)}$ & 1 & $P r_{\text {sig }}$ & \\
\hline EVs & - & 2 & $P r_{\text {sig }}$ & \multirow{5}{*}{$\mathrm{CO}_{2}$ reduction } \\
\hline ESS & - & 2 & $P r_{\text {sig }}$ & \\
\hline $\mathrm{DR}$ & - & 1 & $P r_{\text {sig }}$ & \\
\hline DG * & - & 2 & $P r_{\text {sig }}^{\circ}$ & \\
\hline Market sell & - & 2 & $P r_{s i g}$ & \\
\hline
\end{tabular}

*DG and external suppliers' variables.

The rules, which may improve fitness condition, namely profit maximization are: signal EVs and/or ESS to discharge if the system marginal cost is higher than the contracted price with code -1 , signal EVs and/or ESS to charge if the system marginal cost is lower than the contracted price (code 1), signal DR to use if the system marginal cost is higher than the DR contracted price plus the load income (code 1), signal DR with code 0 if the system marginal cost is lower than the contracted price plus the load income, signal market energy offer to increase if the system marginal cost is lower than the market price (code 1), signal market energy offers with code 0 if the system marginal cost is higher than the market price.

The rules which may help to find solutions with lower $\mathrm{CO}_{2}$ emissions are: signal V2G/ESS resources with code 0 and a signaling probability of $50 \%$. This will set the variables to 0 in the following iteration; signal DR use to increase by using code 1 and a signaling probability of $50 \%$; signal suppliers and DG units that generate $\mathrm{CO}_{2}$ emissions with a probability of $50 \%$ and with code 0 ; signal market energy offers with code 0 and a probability of $50 \%$, to avoid more energy demand and consequently more $\mathrm{CO}_{2}$ emissions. 


\section{Case Study}

The proposed methodology was tested using a case study implemented on a MV $30 \mathrm{kV}$ distribution network with 233 buses. This is part of a real network from Vila Real in Portugal. A reconfiguration was performed to the original mostly meshed network using the software developed in [41] to obtain the radial configuration presented in Figure 1. This single-line diagram does not represent the actual geographical design.

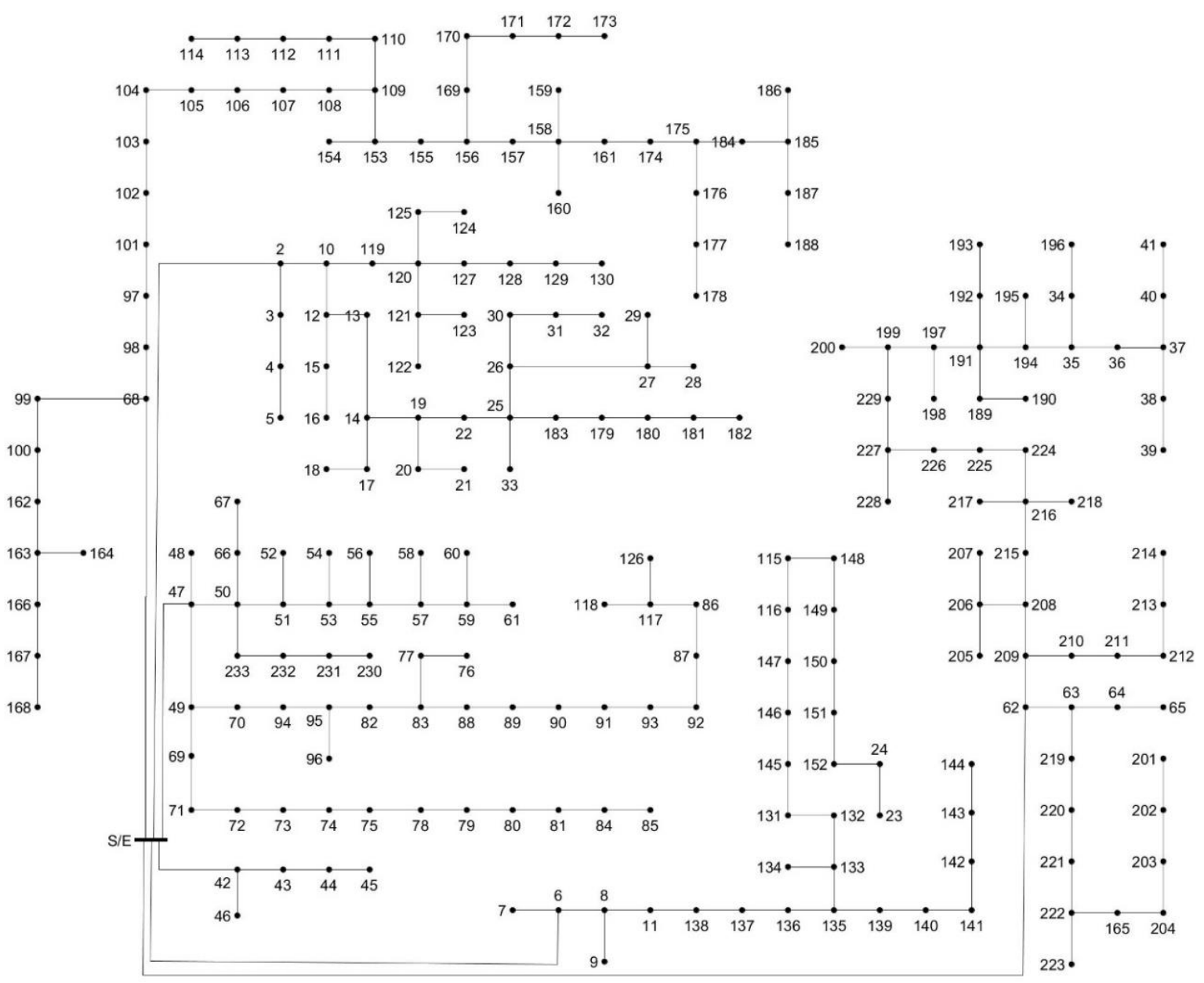

Figure 1. Vila Real 233-bus kV medium-voltage (MV) network.

The work was developed in MATLAB R2014a 64 bits (MathWorks, Natick, MA, USA) using a computer equipped with one Intel Xeon E5-1650 processor (Intel Corporation, Santa Clara, CA, USA) and 10 GB of RAM running Windows 8.1. The parallel platform is implemented in MATLAB distributed computing, i.e., a total of six computers with 42 cores. The single-core tests are done on the mentioned workstation.

\subsection{Scenario Description}

The regular demand (without EVs) was forecasted for 2020 and 2050, taking into account the published results [3,42,43]. According to [3], the consumption will rise by $4 \%$ and $29 \%$ for 2020 and 2050, respectively, in comparison with 2010. Hence, an updated consumption curve for 2020 and 2050 were obtained from the base consumption data available for this grid. Taken into account the actual population $(21,000)$ in Vila Real city, the total number of regular vehicles was estimated using the expected growth/decay rate and the vehicle rate per person. According to the penetration rate of EVs provided by [43], the total number of EVs was set to 1540 for 2020 and 5080 for 2050. The EVs' 
scenarios were created using EVeSSi tool (GECAD, Porto, Portugal) [44]. The charging and discharging efficiency considered was $80 \%$ for 2020 and $90 \%$ for 2050. According to [3], the forecasted penetration of renewable generation in Portugal will amount to 49\% and 77\% in 2020 and 2050, respectively. Photovoltaic capacity installation is expected to be larger than wind installation in the future [3]. Moreover, it was considered that the capacity of the combined heat and power (CHP) increased by two-fold in 2050.

Table 2 shows the data for the developed scenario, taken into account the mentioned forecasts. The considered prices took into account the levelized generation costs presented in [42]. In reality the prices correspond to the cost that the VPP has to pay to buy energy from the respective DER' owner, except from the charge of ESS and EVs, where the owners pay to the VPP instead, therefore contributing to the income in Equation (1) (see Section 2.1). The loads also pay to the VPP and the price varies as can be seen in Table 2 depending on the contract (consumer type).

Table 2. Energy resource data. CHP: combined heat and power; and PV: photovoltaic.

\begin{tabular}{|c|c|c|c|c|c|c|c|}
\hline \multirow{2}{*}{\multicolumn{2}{|c|}{ Energy Resources }} & \multicolumn{2}{|c|}{$\begin{array}{c}\text { Availability (MW) } \\
\text { Minimum-Maximum }\end{array}$} & \multicolumn{2}{|c|}{$\begin{array}{c}\text { Prices (m.u./kWh) } \\
\text { Minimum-Maximum }\end{array}$} & \multicolumn{2}{|c|}{$\begin{array}{l}\text { Numbers of } \\
\text { Units }\end{array}$} \\
\hline & & 2020 & 2050 & 2020 & 2050 & 2020 & 2050 \\
\hline \multicolumn{2}{|c|}{ Biomass } & $0-0.20$ & $0-0.50$ & 0.15 & 0.15 & 1 & 1 \\
\hline \multicolumn{2}{|c|}{ CHP } & $0-1.80$ & $0-4.00$ & 0.10 & 0.10 & 3 & 3 \\
\hline \multicolumn{2}{|c|}{ Small hydro } & $0-1.83$ & $0-2.52$ & 0.13 & 0.13 & 1 & 1 \\
\hline \multicolumn{2}{|c|}{ PV } & 0-1.04 & $0-3.75$ & 0.20 & 0.20 & 81 & 81 \\
\hline \multicolumn{2}{|c|}{ Wind } & $0.20-1.15$ & $0.38-2.22$ & 0.12 & 0.12 & 48 & 48 \\
\hline \multicolumn{2}{|c|}{ External supplier } & $0-10.00$ & $0-15.00$ & 0.11 & 0.11 & 1 & 1 \\
\hline \multirow{2}{*}{ Storage } & Charge & $0-0.25$ & $0-2.00$ & 0.12 & 0.12 & \multirow{2}{*}{4} & \multirow{2}{*}{8} \\
\hline & Discharge & $0-0.25$ & $0-2.00$ & 0.18 & 0.18 & & \\
\hline \multirow{2}{*}{$\mathrm{EV}$} & Charge & $0-8.33$ & $0-31.29$ & 0.14 & 0.14 & \multirow{2}{*}{1540} & \multirow{2}{*}{5080} \\
\hline & Discharge & $0-7.31$ & $0-29.21$ & 0.19 & 0.19 & & \\
\hline $\mathrm{DR}$ & Red & $0-1.06$ & $0-1.31$ & 0.07 & 0.07 & 89 & 89 \\
\hline \multicolumn{2}{|c|}{ Load } & $6.59-14.76$ & $8.20-18.34$ & $0.09-0.15$ & $0.09-0.15$ & 162 & 162 \\
\hline \multicolumn{2}{|c|}{ Market } & $0-4.00$ & $0-4.00$ & $0.08-0.10$ & $0.08-0.10$ & 1 & 1 \\
\hline
\end{tabular}

It is assumed that the VPP is responsible to manage the distribution network and all the energy resources aiming to maximize profit and minimize $\mathrm{CO}_{2}$. It is expected that solutions with higher profits are also those with higher $\mathrm{CO}_{2}$ emissions. The 14,000 network consumers are aggregated by bus totaling 162 aggregated bus-loads. In addition, 89 of the 162 aggregated loads offer DR possibility. The DG units are also aggregated by bus and by type as can be seen in Table 2 . The external supplier located in the substation represents the energy imported from the main grid and is modeled with a $10 \mathrm{MW}$ contract for 2020 and $15 \mathrm{MW}$ for 2050. The EVs are considered individually, increasing 3.3 times in 2050 when compared with 2020. The maximum energy that VPP can export is depicted in the table as the "market" resource, i.e., a maximum of $4 \mathrm{MW}$.

Table 3 presents the energy supplier (main grid) and the $\mathrm{CHP} \mathrm{CO}_{2}$ emission rate taken into account the values presented in [3,45]. A considerable reduction of CHP emission rate in the 2050 scenario from 2020 is considered.

Table 3. Carbon dioxide $\left(\mathrm{CO}_{2}\right)$ parameters of the scenarios.

\begin{tabular}{ccc}
\hline Scenario & Supplier $\mathbf{C O}_{2}$ Emissions $\left(\mathbf{k g C O}_{\mathbf{2}} \mathbf{\text { MWh} )}\right.$ & $\mathbf{C H P ~ C O}_{\mathbf{2}}$ Emissions $(\mathbf{k g C O} / \mathbf{M W h})$ \\
\hline 2020 & 190 & $444-963$ \\
2050 & 20 & 230 \\
\hline
\end{tabular}


Table 4 presents the parameters founded by empirical testing. W-PSO is run independently using the weighted sum approach (see Section 3), namely with a set of 100 weights randomly generated for the two objectives, profit and emissions. In the end, a Pareto front algorithm determines the Pareto front in W-PSO, while NSGA-II and MOPSO obtain the Pareto front in a single run. In fact, the results shown in the following sections for scenarios 2020 (Section 4.2) and 2050 (Section 4.3) have been obtained using a random trial. Previously, a robustness test was performed to understand the results variability for the implemented algorithms. In Section 4.4 this test is described, which verified that the variation of the solutions between trials was quite small. Any random trial should fall within those depicted ranges with a high probability.

Table 4. Metaheuristics-based approaches parameters. W-PSO: weighted particle swarm optimization; MOPSO: multi-objective particle swarm optimization; and NSGA-II: non-dominated sorting genetic algorithm II.

\begin{tabular}{|c|c|c|c|c|}
\hline \multicolumn{2}{|c|}{ Parameters } & W-PSO & MOPSO & NSGA-II \\
\hline \multicolumn{2}{|c|}{ \#Individuals } & 10 & 50 & 50 \\
\hline \multicolumn{2}{|c|}{ Initial solution } & \multicolumn{3}{|c|}{ Random between variable bounds } \\
\hline \multicolumn{2}{|c|}{ Repository size } & - & 100 & - \\
\hline \multicolumn{2}{|c|}{ Number of divisions } & - & 30 & - \\
\hline \multicolumn{2}{|c|}{ Inertia weight } & \multirow{4}{*}{$\begin{array}{l}\text { Gaussian mutation } \\
\text { weights between } 0 \text { and } 1\end{array}$} & 0.73 & - \\
\hline \multicolumn{2}{|c|}{ Acceleration coefficient } & & 1.50 & - \\
\hline \multicolumn{2}{|c|}{ Cooperation coefficient } & & 1.50 & - \\
\hline \multicolumn{2}{|c|}{ Perturbation coefficient } & & 0.97 & - \\
\hline \multicolumn{2}{|c|}{ Mutation learning parameter } & \multicolumn{2}{|l|}{0.20} & - \\
\hline \multicolumn{2}{|c|}{ Mutation rate } & - & 0.50 & 0.10 \\
\hline \multicolumn{2}{|c|}{ Crossover rate } & - & - & 0.70 \\
\hline \multicolumn{2}{|c|}{ Crossover range } & - & - & 0.30 \\
\hline \multicolumn{2}{|c|}{ Maximum velocities } & \multirow{2}{*}{\multicolumn{2}{|c|}{ Velocity clamping factor equation }} & - \\
\hline Minim & $\mathrm{n}$ velocities & & & - \\
\hline \multicolumn{2}{|c|}{ Velocity clamping factor } & 1 & 1 & - \\
\hline \multirow{2}{*}{ Stopping criteria } & Minimum iterations & 500 & - & - \\
\hline & Maximum iterations & & & \\
\hline \multicolumn{2}{|c|}{ Maximum positions } & \multicolumn{3}{|c|}{ Equal to the upper bounds of the variables } \\
\hline \multicolumn{2}{|c|}{ Minimum positions } & \multicolumn{3}{|c|}{ Equal to the lower bounds of the variables } \\
\hline
\end{tabular}

\subsection{Results-Scenario Portugal 2020}

This section presents the results that have been studied for the scenario 2020. Figure 2 presents the Pareto front achieved in MOPSO, W-PSO and NSGA-II metaheuristics with and without signaling, respectively. The marker represents each obtained NDS. NDS-L (left) and NDS-R (right), represents the NDS with lower emissions and higher profit, respectively. There are some Pareto front regions with more markers representing higher density of NDS. Important to refer that without the use of the signaling method, the only metaheuristic that found solutions without violations was the W-PSO (8 NDS). In contrast, all the NDS found by MOPSO and NSGA-II, had violations, which was caused by the voltage and line limit violations and by insufficient generation. The range of the Pareto front results were greatly reduced when compared with the results obtained using the signaling method. Hence, it is possible to conclude that the signaling method use contributed to a better performance of the three metaheuristics addressed in this paper. In the results with the signaling method, the MOPSO profit 
ranged between 6771 m.u. and 10,058 m.u.; while in the W-PSO, the profit ranged between 6355 m.u. and 10,563 m.u. whereas in the NSGA-II, the profit ranged between 7506 m.u. and 10,006 m.u. In terms of $\mathrm{CO}_{2}$ emissions, the range varies between $64.11 \mathrm{tCO}_{2}$ and $67.79 \mathrm{tCO}_{2}\left(\mathrm{tCO}_{2}\right.$ is equivalent to $1000 \mathrm{~kg}$ $\mathrm{CO}_{2}$ where $\mathrm{t}$ stands for tonne) in MOPSO; $63.67 \mathrm{tCO}_{2}$ and $71.26 \mathrm{tCO}_{2}$ in W-PSO; and $65.02 \mathrm{tCO}_{2}$ and $67.46 \mathrm{tCO}_{2}$ in NSGA-II.

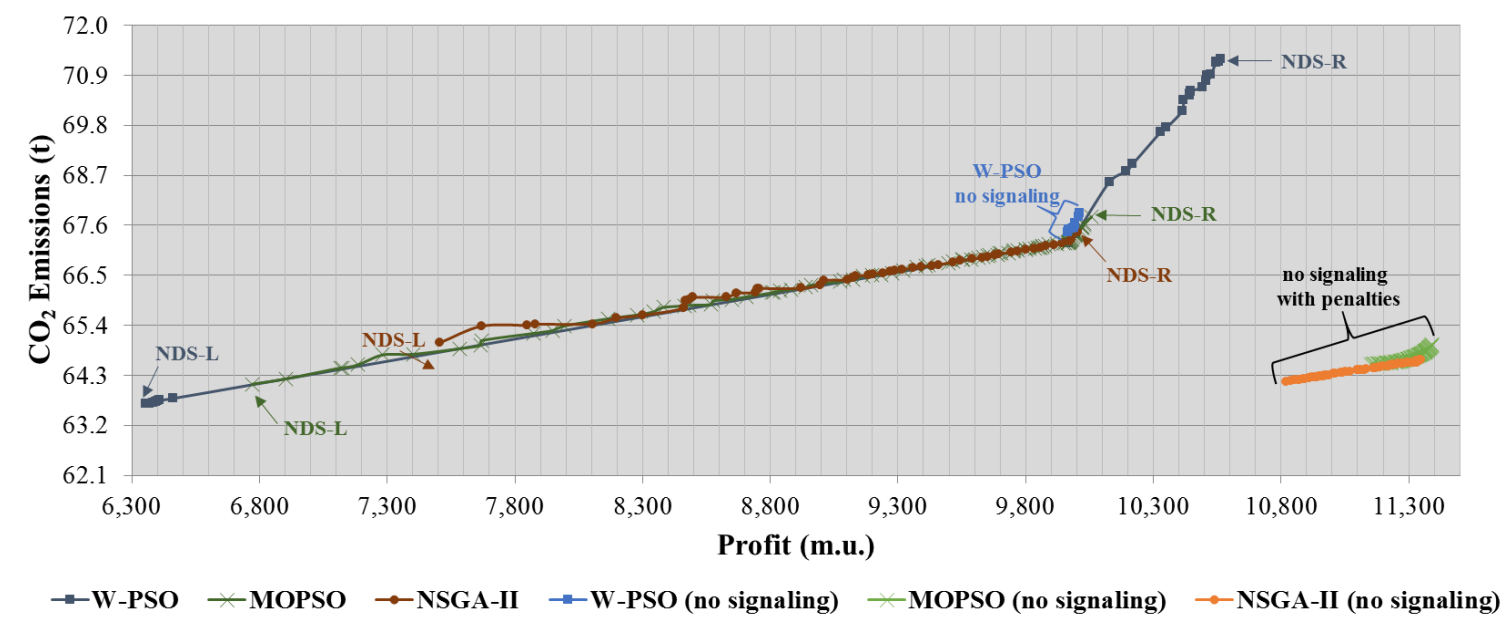

Figure 2. Pareto front for weighted particle swarm optimization (W-PSO), multi-objective particle swarm optimization (MOPSO), and NSGA-II: scenario 2020.

Figures 3 and 4 present the total generation and consumption for two solutions of the Pareto front, namely NDS-L and NDS-R for MOPSO, W-PSO, and NSGA-II method, with the utilization of signaling method. It can be seen that NDS-L uses DR. This enables a significant reduction in the use of power production sources that emit $\mathrm{CO}_{2}$, but will lead to a lower profit due to the need to remunerate customers against the implementation of these measures.

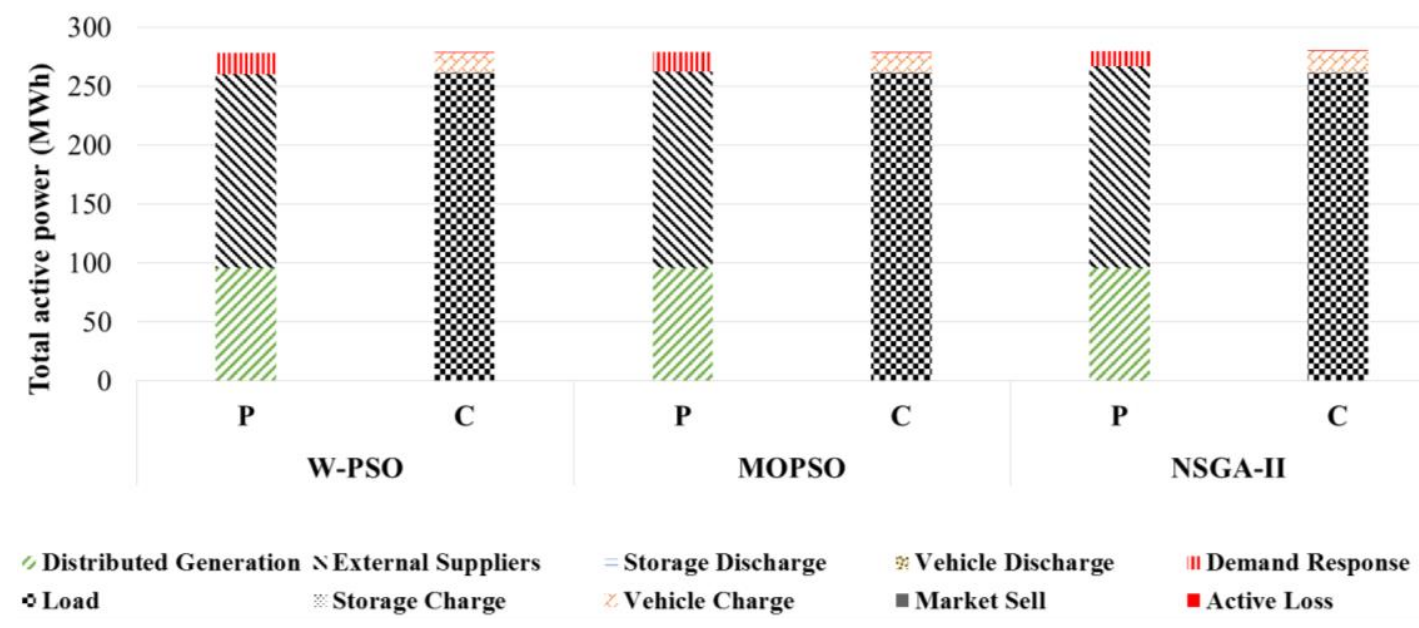

Figure 3. Total generation and consumption: NDS-L (lower emissions): scenario 2020. 


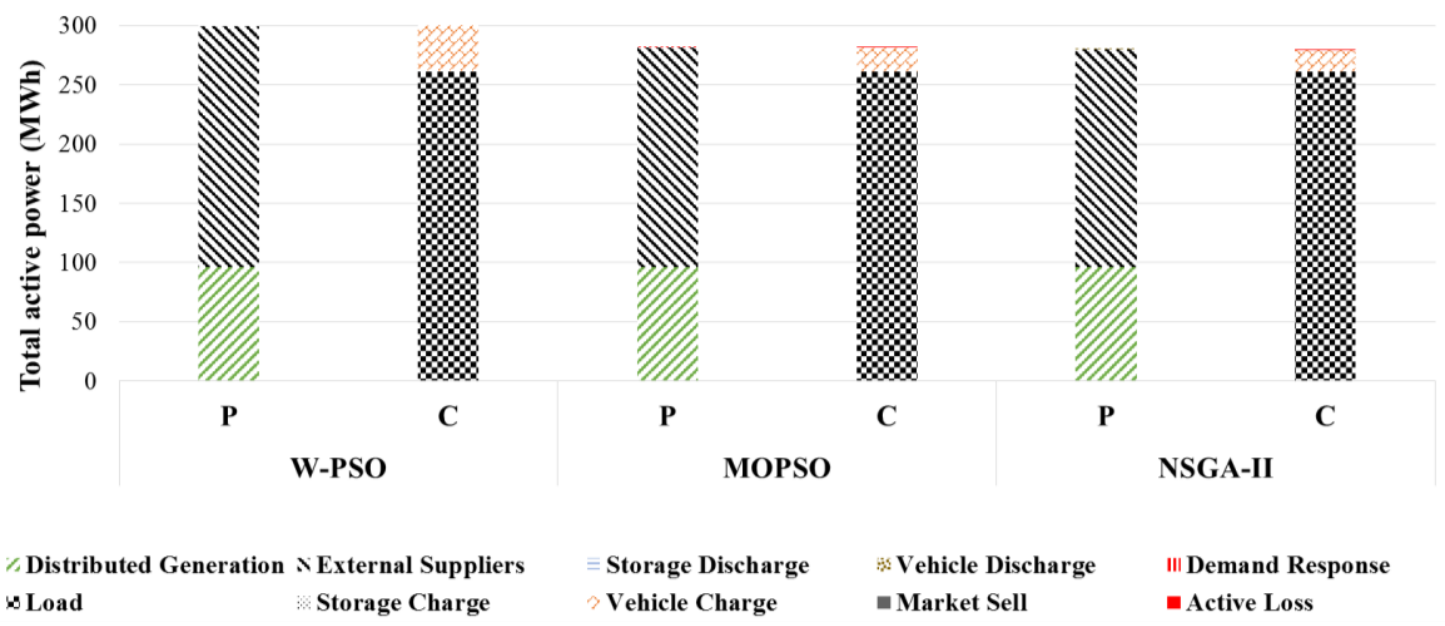

Figure 4. Total generation and consumption: NDS-R (higher profit): scenario 2020.

Table 5 presents the obtained results for each method. The difference between NDS-L and NDS-R were analyzed. The highest profit was obtained in W-PSO (NDS-R solution), i.e., 10,564 m.u. The lowest $\mathrm{CO}_{2}$ emissions was obtained in W-PSO, with $63.67 \mathrm{tCO}_{2}$. The highest difference between NDS- $\mathrm{R}$ and NDS-L in $\mathrm{CO}_{2}$ emissions was achieved with W-PSO, i.e., $7.59 \mathrm{tCO}_{2}$, while the lowest difference was achieved in NSGA-II with $2.44 \mathrm{tCO}_{2}$. In MOPSO the difference was $3.68 \mathrm{tCO}_{2}$. Regarding profit, the highest difference was reported by W-PSO, with 4209 m.u., while NSGA-II presented the lowest difference of the tested methods, with $2500 \mathrm{~m} . \mathrm{u}$. Similarly to the previous scenario, this results indicated that W-PSO method performed better with a higher diversity and better convergence than NSGA-II and MOPSO when signaling method was used. Nevertheless, execution time in W-PSO was much higher than in those methods $(65,100 \mathrm{~s})$. However, in parallel mode the execution time was greatly improved $(16 \times)$ to about 3973 s. In MOPSO this improvement is far less $(3 \times)$ from 7020 $\mathrm{s}$ to $2021 \mathrm{~s}$. In NSGA-II the execution time is $6096 \mathrm{~s}$ in single-core, while the parallel mode did not improved the performance.

Table 5. Scenario 2020 results: total profits and $\mathrm{CO}_{2}$ emissions. NDS: non-dominated solution.

\begin{tabular}{cccccc}
\hline \multicolumn{2}{c}{ Method } & & W-PSO & MOPSO & NSGA-II \\
\hline \multirow{2}{*}{ Indicator } & NDS & & & \\
\hline \multirow{2}{*}{ Profit (m.u.) } & $\mathrm{R}$ & $10,563.49$ & $10,057.91$ & $10,005.88$ \\
\cline { 2 - 5 } & $\mathrm{L}$ & 6354.92 & 6770.93 & 7506.04 \\
\hline \multirow{2}{*}{ Emissions (tCO 2$)$} & $\mathrm{R}$ & 71.26 & 67.79 & 67.46 \\
\cline { 2 - 5 } & $\mathrm{L}$ & 63.67 & 64.11 & 65.02 \\
\hline \multicolumn{2}{c}{ Execution time single core (s) } & 65,100 & 7020 & 6096 \\
\hline \multicolumn{2}{c}{ Execution time (s) } & 3973 & 2021 & - \\
\hline
\end{tabular}

\subsection{Results-Scenario Portugal 2050}

This section presents the obtained results regarding the scenario 2050. Figure 5 presents the Pareto front achieved in MOPSO, W-PSO, and NSGA-II metaheuristics with and without signaling, respectively. 


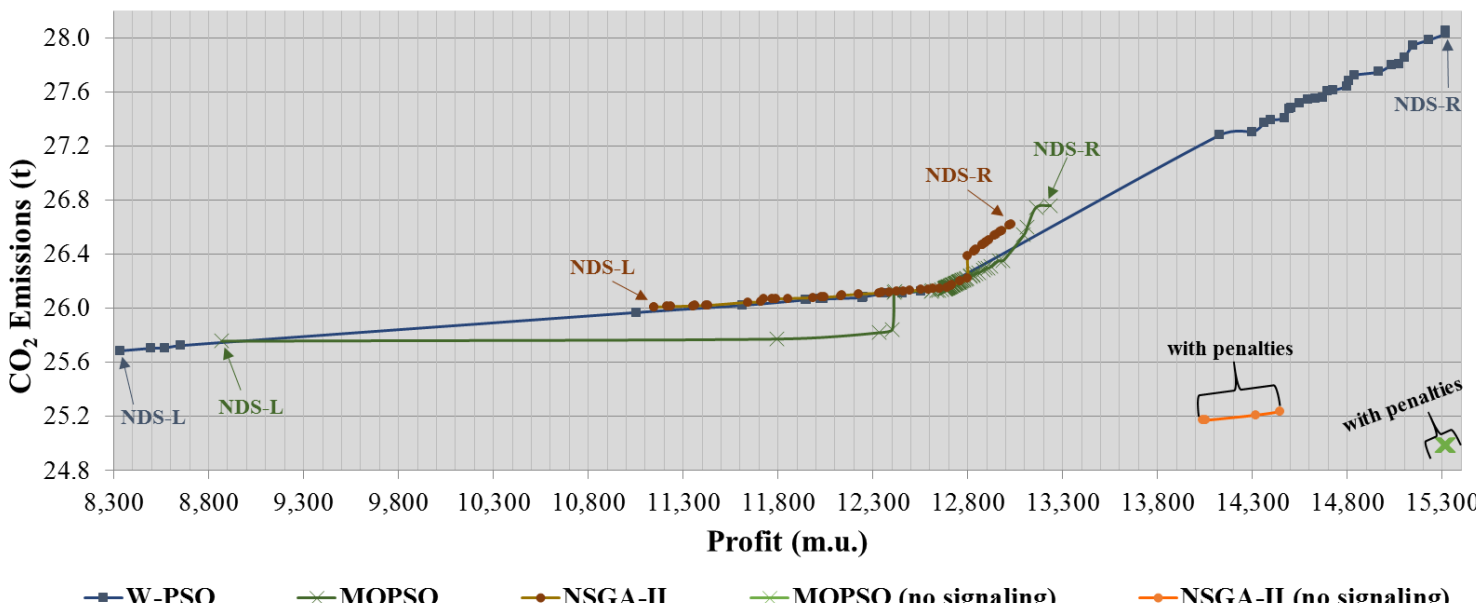

Figure 5. Pareto front for W-PSO, MOPSO, and NSGA-II: scenario 2050.

The marker represents each obtained NDS. NDS-L and NDS-R, represent the NDS with lower emissions and higher profit, respectively. There are some regions of the Pareto front with more markers, representing higher density of NDS. Important to refer that without the use of the signaling method, all the NDS found by the three metaheuristics had violations, caused by voltage limit and line capacity violations and also by insufficient generation. Moreover, the range of the Pareto front was greatly reduced when compared with the results using signaling method. In the results with the signaling method, the MOPSO profit ranged between 8868 m.u. and 13,235 m.u., while in the W-PSO, the profit ranged between $8334 \mathrm{~m} . u$. and $15,320 \mathrm{~m} . \mathrm{u}$. whereas in the NSGA-II, the profit ranged between 11,147 m.u. and 13,030 m.u. In terms of $\mathrm{CO}_{2}$ emissions, the range varies between $25.75 \mathrm{tCO}_{2}$ and $26.76 \mathrm{tCO}_{2}$ in MOPSO; $25.68 \mathrm{tCO}_{2}$ and $28.05 \mathrm{tCO}_{2}$ in W-PSO; and $26.01 \mathrm{tCO}_{2}$ and $26.62 \mathrm{tCO}_{2}$ in NSGA-II.

Figures 6 and 7 present the total generation and consumption for two solutions of the Pareto front, NDS-L and NDS-R, and each of compared methods MOPSO, W-PSO, and NSGA-II with signaling. It can be seen that NDS-L schedules DR whereas NDS-R do not in each method (higher in W-PSO). This use will mean a significant reduction in the generation sources that emit $\mathrm{CO}_{2}$, but will lead to a lower profit due to the need to remunerate customers that use DR measures [46].

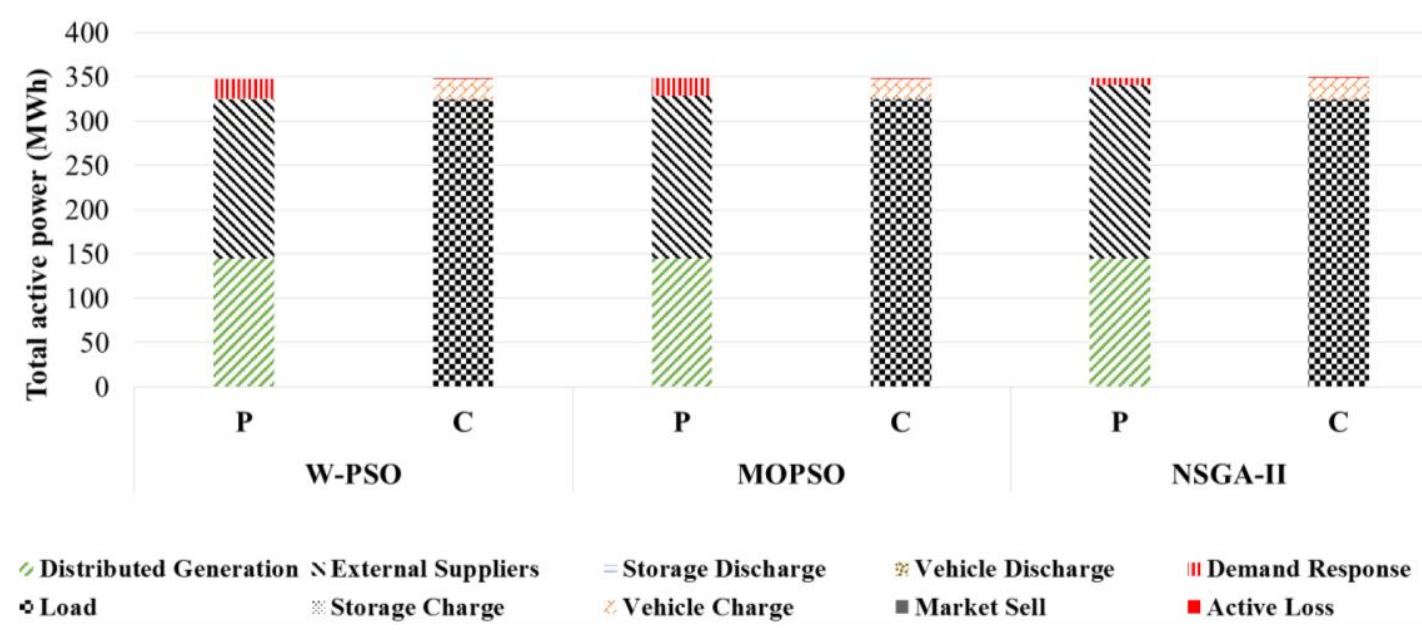

Figure 6. Total generation and consumption: NDS-L (lower emissions): scenario 2050. 


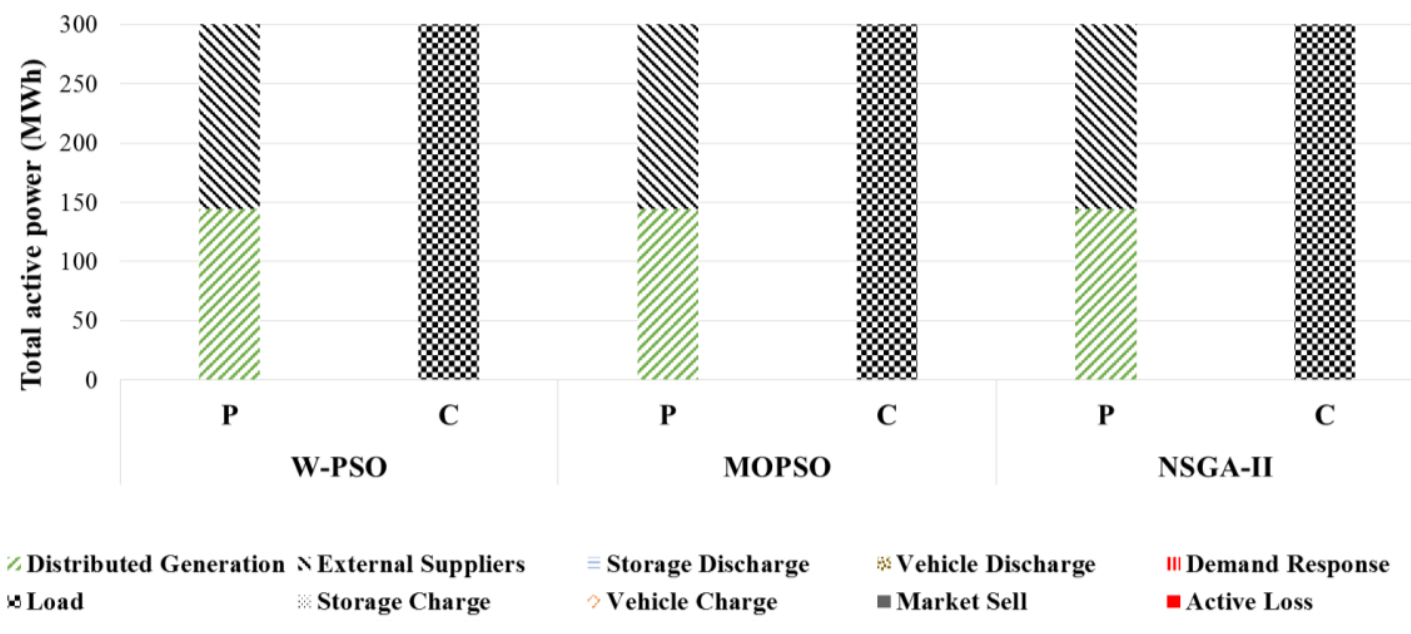

Figure 7. Total generation and consumption: NDS-R (higher profit): scenario 2050.

Table 6 presents the results for each method. The difference between NDS-L and NDS-R can be seen. The highest profit was obtained in W-PSO (NDS-R). The lowest emissions were achieved with W-PSO, i.e., $25.68 \mathrm{tCO}_{2}$. The highest difference between NDS-R and NDS- $\mathrm{L}$ in $\mathrm{CO}_{2}$ emissions was achieved with W-PSO, i.e., $2.37 \mathrm{tCO}_{2}$, while the least difference was achieved in NSGA-II, i.e., $0.61 \mathrm{tCO}$. In MOPSO the difference was $1.01 \mathrm{tCO}_{2}$. Regarding profit, the highest difference was reported by W-PSO, with 6985 m.u., while NSGA-II presented the least difference of the tested methods, with 1883 m.u. Similarly to the previous scenario, this results indicated that W-PSO method performed better with a higher diversity and better convergence than NSGA-II and MOPSO when signaling method was used. Nevertheless, execution time in W-PSO was much higher than those methods.

Table 6. Scenario 2050 results: total profits and emissions.

\begin{tabular}{|c|c|c|c|c|}
\hline \multicolumn{2}{|c|}{ Method } & \multirow{2}{*}{ W-PSO } & \multirow{2}{*}{ MOPSO } & \multirow{2}{*}{ NSGA-II } \\
\hline Indicator & NDS & & & \\
\hline \multirow{2}{*}{ Profit (m.u.) } & $\mathrm{R}$ & $15,319.73$ & $13,235.01$ & $13,029.95$ \\
\hline & $\mathrm{L}$ & 8334.39 & 8868.35 & $11,147.06$ \\
\hline \multirow{2}{*}{ Emissions $\left(\mathrm{tCO}_{2}\right)$} & $\mathrm{R}$ & 28.05 & 26.76 & 26.62 \\
\hline & $\mathrm{L}$ & 25.68 & 25.75 & 26.01 \\
\hline
\end{tabular}

\subsection{Robustness Test}

To confirm the validity of the metaheuristics developed in this paper a robustness testing was computed. This test is necessary because for each run a slightly different Pareto front and solutions can be obtained with these metaheuristics, due to their stochastic nature. For this test each algorithm was run for 100 trials (100 Pareto fronts) in order to confirm that the variability of the obtained results are similar, i.e., robust. Figure 8 presents the Pareto fronts, in two different perspectives: 3D and 2D, obtained in the W-PSO method with the use of signaling method for the 2020 scenario. The W-PSO was chosen here, since this was the method that yielded better solutions comparatively to the other methods, namely NSGA-II and MOPSO (see previous sections). The average standard deviation of the Pareto fronts are 37.25 m.u. $(0.38 \%)$ and 0.039 m.u. (0.05\%) for the profit and emissions, respectively. Despite the low variation, it can be seen that the front deviation tends to be higher (variability) for profitable solutions (Figure 8b). 


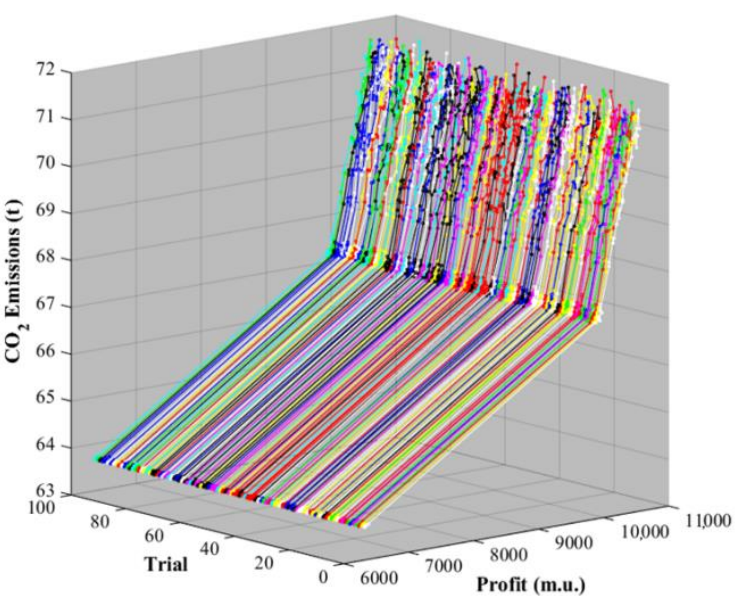

(a)

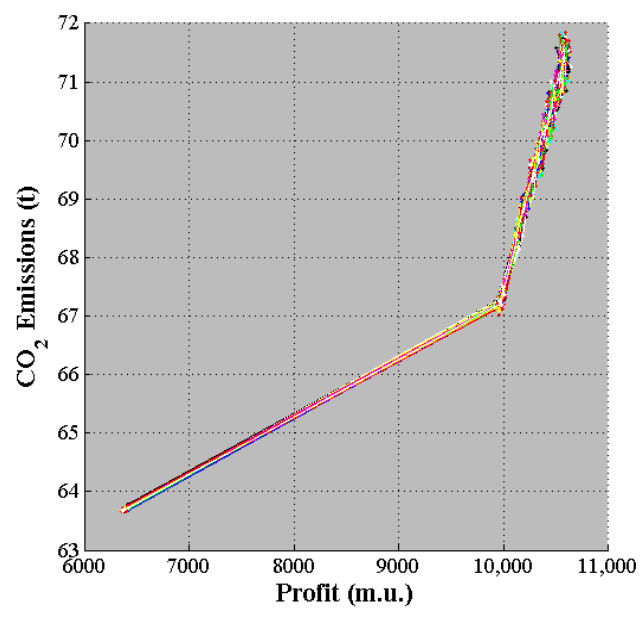

(b)

Figure 8. Variability of the Pareto front obtained in W-PSO method for the 2020 scenario (100 trials).

(a) 3D perspective (all runs); and (b) Pareto front 2D perspective.

\subsection{Parameter Sensitivy Analysis}

A sensitivity analysis experiment has been conducted to understand if W-PSO with signaling was robust enough to variation of essential parameters. The following parameters have been analyzed: number of particles, maximum number of iterations and the number of weight sets. Regarding the number of particles, the experiment has been made with 5, 10 and 20 particles. In what concerns the maximum number of iterations, it has been tested with 200, 500 and 2000 iterations. Finally, the weight sets with 10, 100 (default) and 200 weights. In each test, the remaining parameters of W-PSO have been left unchanged (Table 4). Figure 9 presents the tests made for each parameter as described.

The green line corresponds to the default parameters as tested in the case study (see Table 4). The results suggest that increasing the number of particles and the number of iterations, can improve the Pareto front quality. However, this improvement cannot be deemed significant, except from the number of weight sets. This result suggest that signaling method contributes to W-PSO robustness if the number of weight sets is adequate. The execution times (parallel mode) for each tested case can be seen in the figure. Increasing any of the tested parameters can result in more computational burden. Reducing the number of iterations or the number of weight sets can reduce computational burden, but decrease the Pareto front quality. This degradation is more noticeable when changing the weight sets parameter, e.g., the case with 10 weight sets, the W-PSO obtained 9 NDS with a reduced range of choice regarding profit $/ \mathrm{CO}_{2}$. A trade-off between the Pareto front quality and the experimented parameters is recommend. Therefore, the recommended settings for W-PSO with signaling, based on this experiment are:

- 10 or less particles (a higher number will exponentiallly increase execution times due to the Pareto front selection procedure);

- Between 500 and 2000 iterations (the execution time increases with a higher number of iterations, but it is more reasonable than increasing the number of particles and also more effective, particularly in parallel mode);

- At least 100 weight sets (more weight sets will mean more NDS but much higher computation times). 


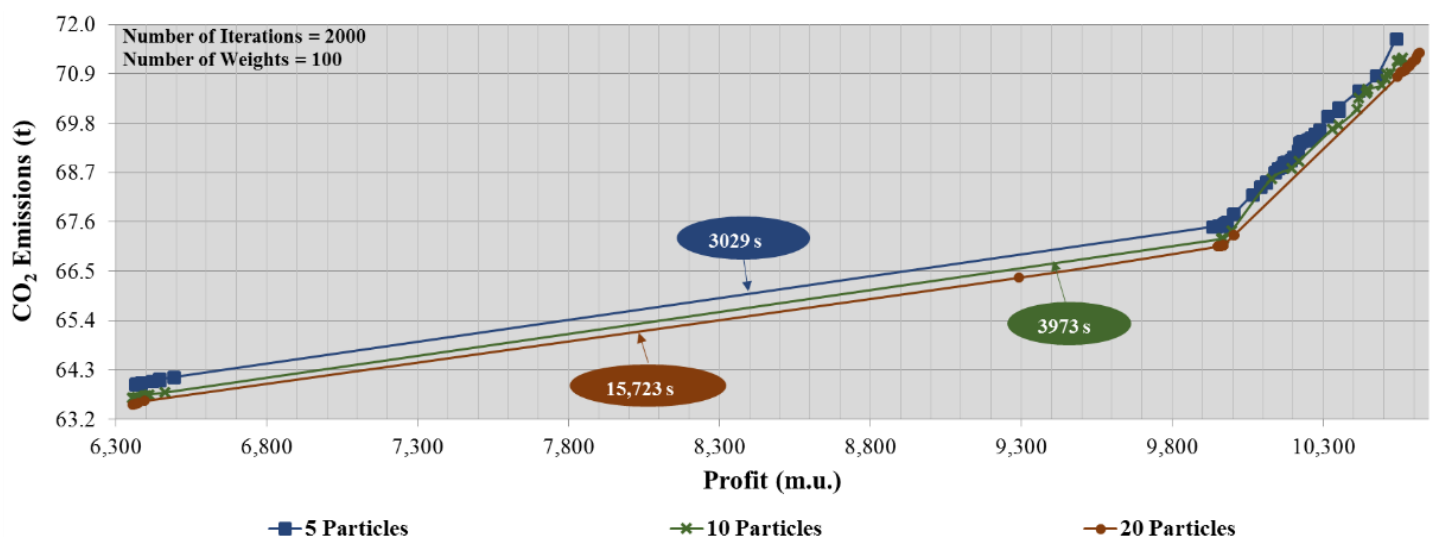

(a)

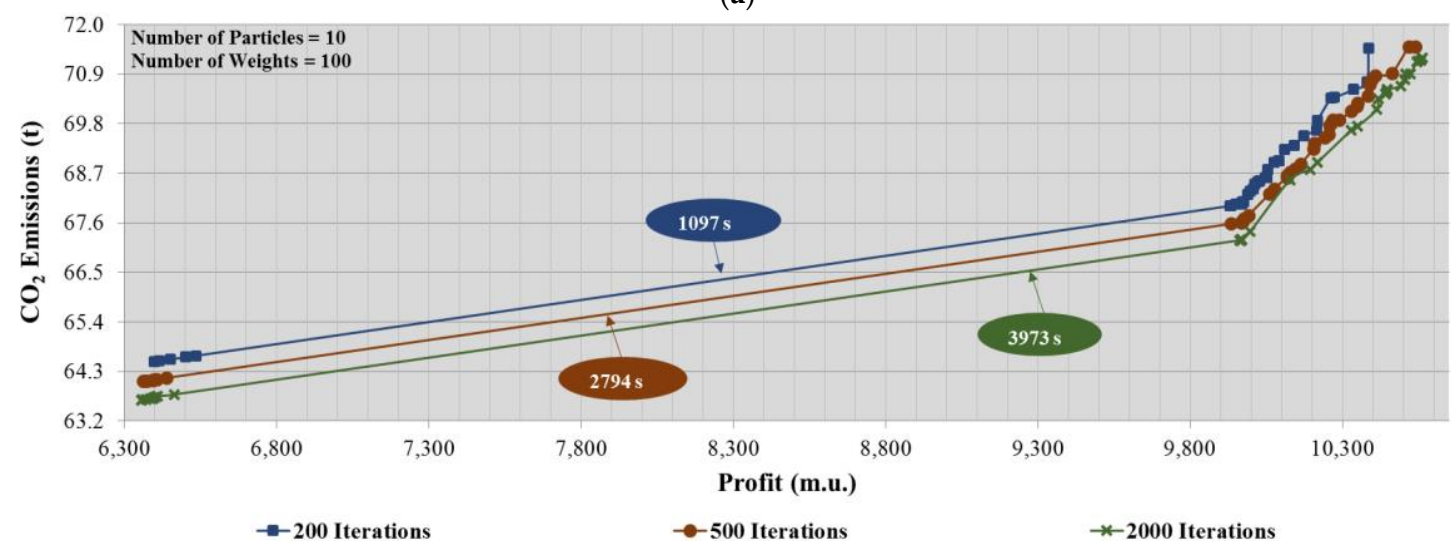

(b)

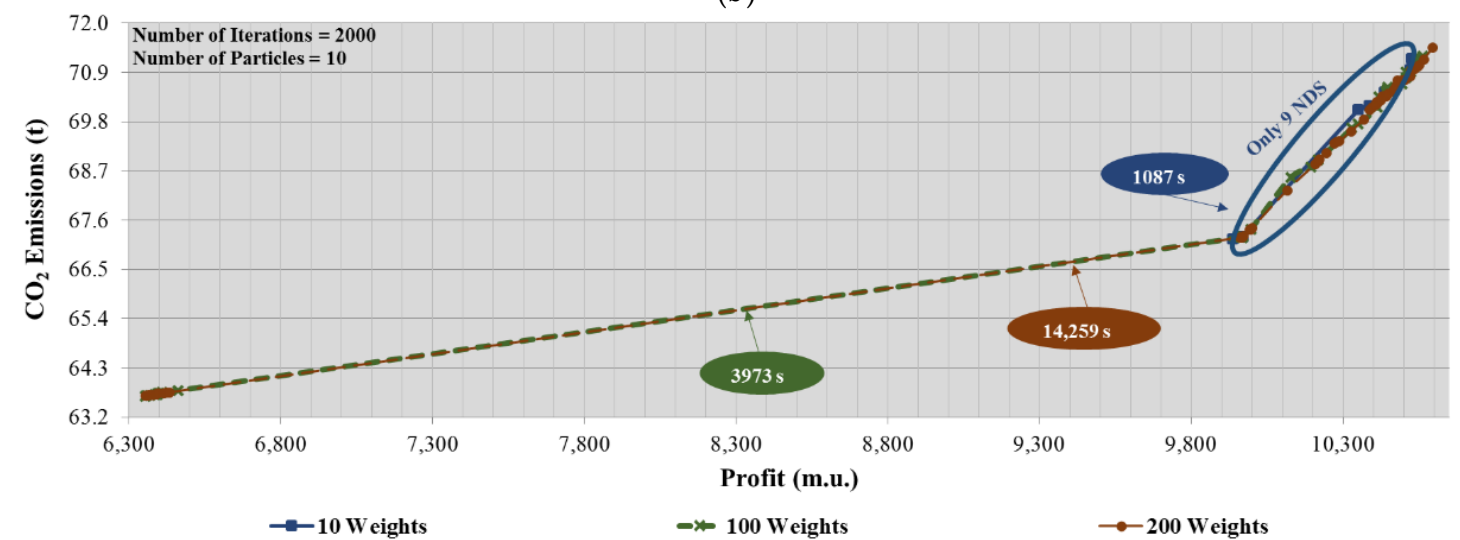

(c)

Figure 9. Sensitivity analysis: (a) number of particles; (b) number of iterations; and (c) number of weight sets.

\section{Conclusions}

This paper presented a study regarding ERM in smart grid with multi-objective goals, namely the VPP profit and $\mathrm{CO}_{2}$ emissions. The metaheuristics implemented to tackle the ERM large-scale optimization are the weighted particle swarm optimization (W-PSO), multi-objective particle swarm optimization (MOPSO), and NSGA-II. A comparison between these methods was made using the signaling method adapted to the multi-objective problem. To validate the proposal, two realistic scenarios were developed using as basis a real distribution grid from Vila Real in Portugal. Several heterogeneous DERs managed by a VPP were considered in the grid. The considered characteristics of 
the cases studied took into account several research work and forecasts available in the literature for 2020 and 2050.

The findings achieved in this paper allowed to understand that the signaling method greatly improved the diversity and convergence of the Pareto front in the metaheuristics-based approaches. In addition, constraints violations were easily avoided with signaling when compared with the standard approaches counterpart, which presented a high number of infeasibilities in both scenarios. This enable to conclude that, the use signaling leverages the better performance of the addressed methods in the ERM as it was demonstrated previously in single-objective problem [23]. In this case, the metaheuristic that featured better results was W-PSO, however, the execution time was higher, when compared with the NSGA-II and MOPSO, mainly in single-core. Therefore, a tradeoff between Pareto front quality and execution time seems to be important as well as considering parallel implementation, which demonstrated to be relevant in this work.

It was verified that $\mathrm{CO}_{2}$ emissions between 2020 and 2050 may drastically reduce if renewable share increases according to available projections. EVs will certainly contribute to increase the average load consumption, despite other loads consumption decrease with energy efficiency improvements. This study also reveals that pursuing $\mathrm{CO}_{2}$ goals reduction within next decades may lose its significance for electricity entities and other similar players. Indeed, the results showed that the profits are substantially affected in exchange for little emission in 2050.

\section{Nomenclature}

Indices

$\begin{array}{ll}I & \text { Index of DG units } \\ t & \text { Index of time periods } \\ L & \text { Index of loads } \\ S & \text { Index of external suppliers } \\ V & \text { Index of EVs } \\ E & \text { Index of ESSs } \\ M & \text { Index of energy buyers }\end{array}$

Sets

$\Omega_{D G}^{d}$

Set of DG units with $\mathrm{CO}_{2}$ emissions

$\Omega_{S P}^{e}$

Set of suppliers with $\mathrm{CO}_{2}$ emissions

Parameters

\begin{tabular}{|c|c|}
\hline$N_{D G}$ & Total number of distributed generators \\
\hline$N_{L}$ & Total number of loads \\
\hline$N_{S T}$ & Total number of storage units \\
\hline$N_{S}$ & Total number of external suppliers \\
\hline$N_{V}$ & Total number of EVs \\
\hline$N_{E}$ & Total number of ESSs \\
\hline$N_{M}$ & Total number of energy buyers \\
\hline$c_{\text {Discharge }(V, t)}$ & Discharging cost of EV $V$ in period $t$ (m.u.) \\
\hline$c_{\text {Discharge }(E, t)}$ & Discharging cost of ESS $E$ in period $t$ (m.u.) \\
\hline$c_{D G(I, t)}$ & Generation price of DG unit $I$ in period $t$ (m.u.) \\
\hline$c_{G C P(I, t)}$ & Generation curtailment power price of DG unit $I$ in period $t$ (m.u.) \\
\hline$c_{N S D(L, t)}$ & Non-supplied demand price of load $L$ in period $t$ (m.u.) \\
\hline
\end{tabular}




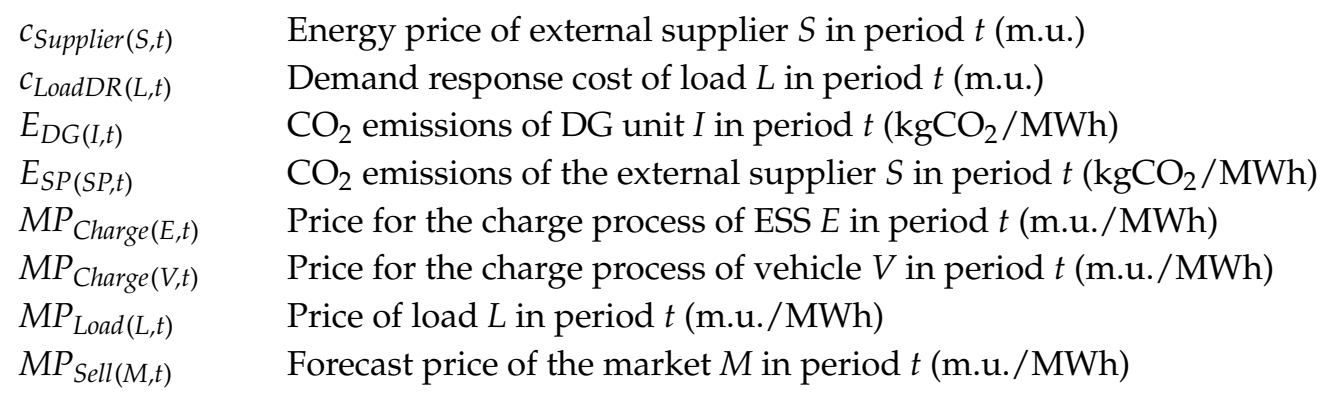

Variables

$\begin{array}{ll}P_{D G(I, t)} & \text { Active power generation of } I \text { unit in period } t(\mathrm{MW}) \\ P_{\text {Supplier }(S, t)} & \text { Active power generation of the external supplier } S \text { in period } t(\mathrm{MW}) \\ P_{\text {LoadDR }(I, t)} & \text { Demand response program active power activated for load } L \text { in period } t(\mathrm{MW}) \\ P_{\text {Charge }(E, t)} & \text { Power charge of ESS unit } E \text { in period } t(\mathrm{MW}) \\ P_{\text {Charge }(V, t)} & \text { Power charge of EV } V \text { in period } t(\mathrm{MW}) \\ P_{\text {Discharge }(E, t)} & \text { Power discharge of ESS unit } E \text { in period } t(\mathrm{MW}) \\ P_{\text {Discharge }(V, t)} & \text { Power discharge of EV } V \text { in period } t(\mathrm{MW}) \\ P_{\text {NSD }(L, t)} & \text { Non-supplied demand for load } L \text { in period } t(\mathrm{MW}) \\ P_{G C P(I, t)} & \text { Generation curtailment power in DG unit } I \text { in period } t(\mathrm{MW}) \\ P_{\text {Load }(L, t)} & \text { Active power demand of load } L \text { in period } t(\mathrm{MW}) \\ \text { Em } & \text { Total emissions } C \mathrm{O}_{2}(\mathrm{~kg}) \\ \text { In } & \text { Total income (m.u.) } \\ \text { OC } & \text { Total operation cost (m.u.) }\end{array}$

Acknowledgments: The present work was done and funded in the scope of the following projects: Horizon 2020 DREAM-GO Project Marie Sklodowska-Curie grant agreement no 641794; UID/EEA/00760/2013, and SFRH/BD/87809/2012 funded by FEDER Funds through COMPETE program and by National Funds through FCT. Authors appreciate the network data supplied by EDP Distribuição, S.A. The original network was simplified to suit the objective of the proposed contribution in this paper.

Author Contributions: All the authors have contributed to the preparation of this manuscript. João Soares and Zita Vale has developed the formulation of the optimization model and supervised the work. In addition, João Soares wrote the paper, developed MOPSO and the signaling method for metaheuristics, and analyzed the results. Nuno Borges has helped to prepare the case study and run several simulations, and created several figures in the manuscript. P.B. de Moura Oliveira has contributed to MOPSO benchmark and quality tests, and also improved the paper in several sections.

Conflicts of Interest: The authors declare no conflict of interest.

\section{Appendix A}

A binary variable, $X_{D G(I, t)}$, is necessary to schedule the DG units that have dispatchable nature (set $\Omega_{D G}^{d}$ ). A value of 1 means that the DG unit is operating at period $t$. The maximum and minimum limits for active $\left(P_{D G M a x L i m i t(I, t)}\right)(\mathrm{kW})$ and reactive $\left(Q_{D G M a x L i m i t(I, t)}\right)$ power of each DG unit $I$ in each period $t$ can be formulated as in Equations (A1) and (A2):

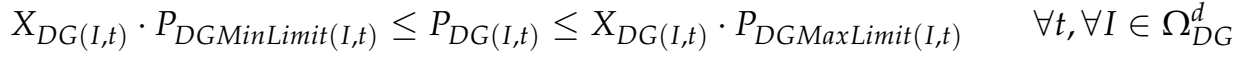

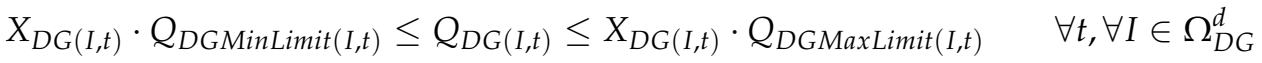

Non-dispatchable DG is formulated in Equation (A3). The $P_{D G F o r e c a s t(I, t)}$ is an input parameter representing the forecast of non dispatchable DG $I$ in period $t(\mathrm{~kW})$. Non-dispatchable DG units are represent by the set $\Omega_{D G}^{n d}$ :

$$
P_{D G(I, t)}=P_{D G F o r e c a s t}(I, t) \quad \forall t, \forall I \in \Omega_{D G}^{n d}
$$


The upstream supplier maximum limit in each period $t$ regarding active power in Equation (A4) and reactive power in Equation (A5) is formulated bellow. The maximum and minimum limits for active and reactive power of each supplier $S$ are inputs represented by $P_{S \operatorname{MaxLimit}(I, t)}(\mathrm{kW})$ and $Q_{S M a x L i m i t(I, t)}(\mathrm{kvar})$ :

$$
\begin{array}{cc}
X_{\text {Supplier }(S, t)} \cdot P_{\text {SMinLimit }(S, t)} \leq P_{\text {Supplier }(S, t)} \leq X_{\text {Supplier }(S, t)} \cdot P_{\text {SMaxLimit }(S, t)} & \forall t, \forall S \\
X_{\text {Supplier }(S, t)} \cdot Q_{\text {SMinLimit }(S, t)} \leq Q_{\text {Supplier }(S, t)} \leq X_{\text {Supplier }(S, t)} \cdot Q_{S \text { MaxLimit }(S, t)} & \forall t, \forall S
\end{array}
$$

The constraints for the ESS (batteries) are described below. The ESS charge and discharge cannot be simultaneous as represented by Equation (A6). Therefore, two binary variables, namely $X_{E S S(E, t)}$ for discharging and $Y_{E S S(E, t)}$ for charging guarantee this condition for each ESS in each period $t$ :

$$
X_{E S S(E, t)}+Y_{E S S(E, t)} \leq 1 \quad \forall t, \forall E
$$

The maximum battery balance for each ESS can be formulated in Equation (A7). The variable $E_{S t o r e d}(E, t)$ represents the energy stored in ESS $E$ in period $t(\mathrm{kWh})$. The $\Delta t$ parameter converts power to energy, i.e., for $t=1 \mathrm{~h}, \Delta t=1, t=30 \mathrm{~min}, \Delta t=0.5$. The parameters $\eta_{c(E)}$ and $\eta_{d(E)}$ corresponds to charging and discharging efficiency of ESS $E(\%)$, respectively:

$$
\begin{gathered}
E_{\text {Stored }(E, t)}=E_{\text {Stored }(E, t-1)}+ \\
\eta_{c(E)} \cdot P_{\text {Charge }(E, t)} \cdot \Delta t-\frac{1}{\eta_{d(E)}} \cdot P_{\text {Discharge }(E, t)} \cdot \Delta t \quad \forall t, \forall E
\end{gathered}
$$

The maximum discharge limit for each ESS can be represented by Equation (A8), where $P_{\text {DischargeLimit }(E, t)}$ is an input regarding the maximum discharge rate of ESS $E$ in period $t(\mathrm{~kW})$. Similarly, Equation (A9) represents the maximum charge limit for each ESS $E$ in each period $t$, where $P_{\text {ChargeLimit }(E, t)}$ is an input parameter $(\mathrm{kW})$ :

$$
\begin{array}{cc}
P_{\text {Discharge }(E, t)} \leq P_{\text {DischargeLimit }(E, t)} \cdot X_{E S S(E, t)} \quad \forall t, \forall E \\
P_{\text {Charge }(E, t)} \leq P_{\text {ChargeLimit }(E, t)} \cdot Y_{E S S(E, t)} \quad \forall t, \forall E
\end{array}
$$

The maximum energy stored in each ESS $E$ for each period $t$ can be represented by Equation (A10), variable $E_{S t o r e d(E, t)}$, and $E_{B a t C a p(E)}$ is an input parameter representing the maximum battery capacity of ESS E (kWh):

$$
E_{\text {Stored }(E, t)} \leq E_{\text {BatCap }(E)} \quad \forall t, \forall E
$$

The minimum stored energy to be guaranteed at the end of period $t$ can be represented by Equation (A11), where $E_{\text {MinCharge }(E, t)}$ is the minimum energy stored required in ESS $E$ in period $t(\mathrm{kWh})$ :

$$
E_{S t o r e d}(E, t) \geq E_{\operatorname{MinCharge}(E, t)} \quad \forall t, \forall E
$$

The constraints for the EVs are described below. The charge and discharge of each EV is not simultaneous. Two binary variables are needed for each vehicle that can be represented by Equation (A12). Therefore, two binary variables, namely $X_{E V(V, t)}$ for discharging and $Y_{E V(V, t)}$ for charging guarantee this condition for each $\mathrm{EV}$ in each period $t$ :

$$
X_{E V(V, t)}+Y_{E V(V, t)} \leq 1 \quad \forall t, \forall V
$$

The battery balance for each EV $\left(E_{S t o r e d}(V, t)\right)$, Equation (A13), and the energy consumption for period $t$ travel $\left(E_{\operatorname{Trip}(V, t)}\right)$ has to be considered jointly with the energy remaining from the previous 
period $(t-1)$ and the charge/discharge occurred in period $t$. Parameters $\eta_{c(E)}$ and $\eta_{d(E)}$ corresponds to charging and discharging efficiency of EV $V(\%)$, respectively:

$$
\begin{gathered}
E_{\text {Stored }(V, t)}=E_{\text {Stored }(V, t-1)}-E_{\text {Trip }(V, t)}+ \\
\eta_{c(V)} \cdot P_{\text {Charge }(V, t)} \cdot \Delta t-\frac{1}{\eta_{d(V)}} \cdot P_{\text {Discharge }(V, t)} \cdot \Delta t \quad \forall t, \forall V
\end{gathered}
$$

When connected to the grid the vehicle cannot discharge to the grid more than the admissible rate $\left(P_{\text {DischargeLimit }(V, t)}\right)(\mathrm{kW})$. The discharge limit for each EV considering battery discharge rate can be formulated as in Equation (A14):

$$
P_{\text {Discharge }(V, t)} \leq P_{\text {DischargeLimit }(V, t)} \cdot X_{E V(V, t)} \quad \forall t, \forall V
$$

where $P_{\text {DischargeLimit }(V, t)}$ is the maximum active discharge rate of $\mathrm{EV} V$ in period $t(\mathrm{~kW})$.

When connected to the grid the vehicle cannot charge more than the admissible safety rate $\left(P_{\text {Chargelimit }(V, t)}\right)(\mathrm{kW})$. The charge limit for each EV considering battery charge rate can be formulated as in Equation (A15):

$$
P_{\text {Charge }(V, t)} \leq P_{\text {ChargeLimit }(V, t)} \cdot Y_{E V(V, t)} \quad \forall t, \forall V
$$

Another important aspect is the minimum stored energy to be guaranteed at the end of period $t$, which is formulated in Equation (A16). This can be seen as a reserve energy $\left(E_{\text {MinCharge }(V, t)}\right.$, fixed by the EVs' users or estimated by the operator) that can be used for a regular travel or an unexpected travel in each period $t$ :

$$
E_{\text {Stored }(V, t)} \geq E_{\operatorname{MinCharge}(V, t)} \quad \forall t, \forall V
$$

Load demand response program limit, namely direct load control, represented by the variable $P_{\text {LoadDR }(L, t, t)}$, load reduced for load $L$ in period $t$ can be formulated as in Equation (A17). The maximum load reduce is limited by $P_{\text {LoadDRMaxLimit }(L, t)}(\mathrm{kW})$ parameter:

$$
P_{\text {LoadDR }(L, t)} \leq P_{\text {LoadDRMaxLimit }(L, t)} \quad \forall t, \forall L
$$

The market offer may be constrained by a minimum and maximum amount, Equations (A18) and (A19), respectively. $X_{\operatorname{Market}(M, t)}$ is a binary variable that represents the choice of market $M$ in period $t$ :

$$
\begin{array}{ll}
P_{\text {Sell }(M, t)} \leq P_{\text {SellMarketMax }(M, t)} \cdot X_{\text {Market }(M, t)} & \forall t, \forall M \\
P_{\text {Sell }(M, t)} \geq P_{\text {SellMarketMin }(M, t)} \cdot X_{\operatorname{Market}(M, t)} & \forall t, \forall M
\end{array}
$$

\section{References}

1. Denny, E.; O'Malley, M. Wind generation, power system operation, and emissions reduction. IEEE Trans. Power Syst. 2006, 21, 341-347. [CrossRef]

2. Foster, V.; Bedrosyan, D. Understanding $\mathrm{CO}_{2}$ Emissions from the Global Energy Sector; Report 85126; World Bank Group: Washington, DC, USA, 2014.

3. EU Energy, Transport and GHG Emissions Trends to 2050: Reference scenario 2013; European Commission: Luxembourg, 2013.

4. Vojdani, A. Smart Integration. IEEE Power Energy Mag. 2008, 6, 71-79. [CrossRef]

5. Lv, T.; Ai, Q.; Zhao, Y. A bi-level multi-objective optimal operation of grid-connected microgrids. Electr. Power Syst. Res. 2016, 131, 60-70. [CrossRef]

6. Ahmadi, A.; Masouleh, M.S.; Janghorbani, M.; Manjili, N.Y.G.; Sharaf, A.M.; Nezhad, A.E. Short term multi-objective hydrothermal scheduling. Electr. Power Syst. Res. 2014, 121, 357-367. [CrossRef] 
7. Kuroda, K.; Magori, H.; Ichimura, T.; Yokoyama, R.A. hybrid multi-objective optimization method considering optimization problems in power distribution systems. J. Mod. Power Syst. Clean Energy 2015, 3, 41-50. [CrossRef]

8. Zakariazadeh, A.; Jadid, S.; Siano, P. Multi-objective scheduling of electric vehicles in smart distribution system. Energy Convers. Manag. 2014, 79, 43-53. [CrossRef]

9. Aghajani, G.R.; Shayanfar, H.A.; Shayeghi, H. Presenting a multi-objective generation scheduling model for pricing demand response rate in micro-grid energy management. Energy Convers. Manag. 2015, 106, 308-321. [CrossRef]

10. De Moura Oliveira, P.B.; Pires, E.J.S.; Cunha, J.B.; Vrančić, D. Multi-objective particle swarm optimization design of PID controllers. In Distributed Computing, Artificial Intelligence, Bioinformatics, Soft Computing, and Ambient Assisted Living; Springer: Salamanca, Spain, 2009; pp. 1222-1230.

11. Motevasel, M.; Seifi, A.R. Expert energy management of a micro-grid considering wind energy uncertainty. Energy Convers. Manag. 2014, 83, 58-72. [CrossRef]

12. Niknam, T.; Azizipanah-Abarghooee, R.; Narimani, M.R. An efficient scenario-based stochastic programming framework for multi-objective optimal micro-grid operation. Appl. Energy 2012, 99, 455-470. [CrossRef]

13. Motevasel, M.; Seifi, A.R.; Niknam, T. Multi-objective energy management of CHP (combined heat and power)-based micro-grid. Energy 2013, 51, 123-136. [CrossRef]

14. Aghaei, J.; Alizadeh, M.-I. Multi-objective self-scheduling of CHP (combined heat and power)-based microgrids considering demand response programs and ESSs (energy storage systems). Energy 2013, 55, 1044-1054. [CrossRef]

15. Chaouachi, A.; Kamel, R.M.; Andoulsi, R.; Nagasaka, K. Multiobjective Intelligent Energy Management for a Microgrid. IEEE Trans. Ind. Electron. 2013, 60, 1688-1699. [CrossRef]

16. Korkas, C.D.; Baldi, S.; Michailidis, I.; Kosmatopoulos, E.B. Intelligent energy and thermal comfort management in grid-connected microgrids with heterogeneous occupancy schedule. Appl. Energy 2015, 149, 194-203. [CrossRef]

17. Soares, J.; Lobo, C.; Silva, M.; Morais, H.; Vale, Z. Relaxation of non-convex problem as an initial solution of meta-heuristics for energy resource management. In Proceedings of the 2015 IEEE Power \& Energy Society General Meeting, Denver, CO, USA, 2015; pp. 1-5.

18. Soares, J.; Fotouhi Ghazvini, M.A.; Vale, Z.; de Moura Oliveira, P.B. A multi-objective model for the day-ahead energy resource scheduling of a smart grid with high penetration of sensitive loads. Appl. Energy 2016, 162, 1074-1088. [CrossRef]

19. Saber, A.Y.; Venayagamoorthy, G.K. Intelligent unit commitment with vehicle-to-grid-A cost-emission optimization. J. Power Sources 2010, 195, 898-911. [CrossRef]

20. Coello, C.A.C.; Pulido, G.T.; Lechuga, M.S. Handling multiple objectives with particle swarm optimization. IEEE Trans. Evol. Comput. 2004, 8, 256-279. [CrossRef]

21. Soares, J.; Silva, M.; Sousa, T.; Vale, Z.; Morais, H. Distributed energy resource short-term scheduling using Signaled Particle Swarm Optimization. Energy 2012, 42, 466-476. [CrossRef]

22. Soares, J.; Sousa, T.; Morais, H.; Vale, Z.; Canizes, B.; Silva, A. Application-specific modified particle swarm optimization for energy resource scheduling considering vehicle-to-grid. Appl. Soft Comput. 2013, 13, 4264-4280. [CrossRef]

23. Soares, J.; Fotouhi Ghazvini, M.A.; Silva, M.; Vale, Z. Multi-dimensional signaling method for population-based metaheuristics: Solving the large-scale scheduling problem in smart grids. Swarm Evol. Comput. 2016, 29, 13-32. [CrossRef]

24. Navigant Research: Virtual Power Plants. Available online: http://www.navigantresearch.com/research/ virtual-power-plants (accessed on 24 July 2015).

25. Morais, H.; Pinto, T.; Vale, Z.; Praca, I. Multilevel negotiation in smart grids for vpp management of distributed resources. IEEE Intell. Syst. 2012, 27, 8-16. [CrossRef]

26. Vasirani, M.; Kota, R.; Cavalcante, R.L.G.; Ossowski, S.; Jennings, N.R. An agent-based approach to virtual power plants of wind power generators and electric vehicles. IEEE Trans. Smart Grid 2013, 4, 1314-1322. [CrossRef]

27. You, S.; Traeholt, C.; Poulsen, B. A market-based Virtual Power Plant. In Proceedings of the 2009 International Conference on Clean Electrical Power, Capri, Italy, 9-11 June 2009; pp. 460-465. 
28. Thukaram, D.; Wijekoon Banda, H.M.; Jerome, J. A robust three phase power flow algorithm for radial distribution systems. Electr. Power Syst. Res. 1999, 50, 227-236. [CrossRef]

29. Soares, J.; Silva, M.; Vale, Z.; de Moura Oliveira, P.B. Quantum-based PSO applied to hour-ahead scheduling in the context of smart grid management. In Proceedings of the 2015 IEEE Eindhoven PowerTech, Eindhoven, The Netherlands, 29 June-2 July 2015; pp. 1-6.

30. Conejo, A.J.; Carrión, M.; Morales, J.M. Decision Making Under Uncertainty in Electricity Markets; International Series in Operations Research \& Management Science; Springer: Boston, MA, USA, 2010; Volume 153.

31. Talbi, E.-G.; Basseur, M.; Nebro, A.J.; Alba, E. Multi-objective optimization using metaheuristics: Non-standard algorithms. Int. Trans. Oper. Res. 2012, 19, 283-305. [CrossRef]

32. Silva, M.; Morais, H.; Sousa, T.; Vale, Z. Energy resources management in three distinct time horizons considering a large variation in wind power. In Proceedings of the Europe's Premier Wind Energy Annual Event 2013, Vienna, Austria, 4-7 February 2013.

33. Logenthiran, T.; Srinivasan, D.; Khambadkone, A.M.; Aung, H.N. Multiagent system for real-time operation of a microgrid in real-time digital simulator. IEEE Trans. Smart Grid 2012, 3, 925-933. [CrossRef]

34. Fotouhi, M.A.; Soares, J.; Horta, N.; Neves, R.; Castro, R.; Vale, Z. A multi-objective model for scheduling of short-term incentive-based demand response programs offered by electricity retailers. Appl. Energy 2015, 151, 102-118. [CrossRef]

35. Miranda, V.; Keko, H.; Jaramillo, A. EPSO: Evolutionary particle swarms. Stud. Comput. Intell. 2007, 66, 139-167.

36. Rahman, D.F.; Viana, A.; Pedroso, J.P. Metaheuristic search based methods for unit commitment. Int. J. Electr. Power Energy Syst. 2014, 59, 14-22. [CrossRef]

37. Boussaïd, I.; Lepagnot, J.; Siarry, P. A survey on optimization metaheuristics. Inf. Sci. 2013, 237, 82-117. [CrossRef]

38. Deb, K.; Pratap, A.; Agarwal, S.; Meyarivan, T. A fast and elitist multiobjective genetic algorithm: NSGA-II. IEEE Trans. Evol. Comput. 2002, 6, 182-197. [CrossRef]

39. Chaube, A.; Benyoucef, L.; Tiwari, M.K. An adapted NSGA-2 algorithm based dynamic process plan generation for a reconfigurable manufacturing system. J. Intell. Manuf. 2010, 23, 1141-1155. [CrossRef]

40. Mandal, D.; Pal, S.K.; Saha, P. Modeling of electrical discharge machining process using back propagation neural network and multi-objective optimization using non-dominating sorting genetic algorithm-II. J. Mater. Process. Technol. 2007, 186, 154-162. [CrossRef]

41. Dias, F.M.; Canizes, B.; Khodr, H.; Cordeiro, M. Distribution networks planning using decomposition optimisation technique. IET Gener. Transm. Distrib. 2015, 9, 1409-1420. [CrossRef]

42. Levelized Cost and Levelized Avoided Cost of New Generation Resources in the Annual Energy Outlook 2015; U.S. Energy Information Administration: Washington, DC, USA, 2015.

43. Reis, N.M.M. Desenvolvimento de Algoritmos de Controlo de Carregamentos de Veículos Eléctricos Controlados por Energy Box no Local de Consumo. Master's Thesis, Universidade de Coimbra, Coimbra, Portugal, 2011. (In Portuguese).

44. Soares, J.; Canizes, B.; Lobo, C.; Vale, Z.; Morais, H. Electric vehicle scenario simulator tool for smart grid operators. Energies 2012, 5, 1881-1899. [CrossRef]

45. Killip, G. Emission Factors and the Future of Fuel; University of Oxford: Oxford, UK, 2005.

46. Faria, P.; Vale, Z.; Baptista, J. Demand response programs design and use considering intensive penetration of distributed generation. Energies 2015, 8, 6230-6246. [CrossRef]

(C) 2016 by the authors; licensee MDPI, Basel, Switzerland. This article is an open access article distributed under the terms and conditions of the Creative Commons Attribution (CC-BY) license (http://creativecommons.org/licenses/by/4.0/). 